\title{
From Three-Dimensional Space Vision to Prehensile Hand Movements: The Lateral Intraparietal Area Links the Area V3A and the Anterior Intraparietal Area in Macaques
}

\author{
Hiroyuki Nakamura, ${ }^{1,2}$ Tatsuya Kuroda, ${ }^{3}$ Masumi Wakita, ${ }^{4}$ Makoto Kusunoki, ${ }^{5}$ Akemi Kato, ${ }^{4}$ Akichika Mikami, \\ Hideo Sakata, ${ }^{5}$ and Kazuo Itoh ${ }^{1}$ \\ ${ }^{1}$ Department of Anatomy, ${ }^{2}$ Medical Education Development Center, and ${ }^{3}$ Department of Neurosurgery, Gifu University \\ School of Medicine, Gifu 500-8705, Japan, 4 Section of Neurophysiology, Department of Behavioral and Brain Sciences, \\ Primate Research Institute, Kyoto University, Aichi 484-8506, Japan, and 5epartment of Physiology, Nihon University \\ School of Medicine, Tokyo 173-8610, Japan
}

\begin{abstract}
The posterior parietal cortex is included in the dorsal cortical visual pathway underlying the three-dimensional (3-D) visual recognition of space and objects. The neurons in the lateral intraparietal area (LIP) respond visually to the three-dimensional objects, whereas those in the anterior intraparietal area (AIP) respond to hand movements to grasp them. LIP receives visual inputs from V3A, whereas AIP projects to the premotor areas; however, it is not known whether the neurons in LIP project to AIP. We herein investigated the connectional substrates that underlie the transformation of three-dimensional vision to prehensile hand movements in the Japanese monkey (Macaca fuscata). After identifying the three-dimensional visually responsive region in the posterior part of LIP by the unit recordings, we injected a bidirectional tracer, wheat germ agglutinin conju-
\end{abstract}

gated to horseradish peroxidase, into one of the recording sites. We found that LIP receives neuronal projections from V3A and sends axons to AIP. To confirm our findings, we injected several orthograde tracers into V3A and retrograde tracers into AIP in the same hemispheres. We found that the V3A neurons projecting to LIP terminate in the vicinity of the LIP neurons projecting to AIP. The results suggest that the cortical connections of V3A-LIP-AIP in the lateral bank of the intraparietal sulcus play an important role in the visuomotor transformation for prehensile hand movements.

Key words: parietal cortex; V3A; LIP; AIP; intraparietal visual cortex; three-dimensional vision; hand movements; extrastriate visual areas; dorsal stream
Visual information for three-dimensional (3-D) space is transferred from the occipital lobe to the parietal visual areas through the dorsal stream of the cortical visual pathway in primates (Ungerleider and Mishkin, 1982). The dorsal stream is related to the visual control of actions, and the parietal association areas located in the banks of the intraparietal sulcus are supposed to play important roles in both reaching and prehensile hand movements (Mountcastle et al., 1975; Taira et al., 1990; Goodale et al., 1991; Goodale and Milner, 1992; Milner and Goodale, 1993; Sakata et al., 1995; Goodale and Murphy, 1997; Kalaska et al., 1997; Rizzolatti et al., 1997; Caminiti et al., 1998). The neurons related to hand-reaching movements are recorded in parietal areas 5 and 7, including the medial intraparietal area (MIP), the lateral intraparietal area (LIP), and the parieto-occipital area (PO), in addition to the frontal area dorsal premotor area 6 (F2, F7) (Mountcastle et al., 1975; Rizzolatti et al., 1990; Lacquaniti et al., 1995; Johnson et al., 1996, 1997). MIP and the intraparietal

\footnotetext{
Received Jan. 22, 2001; revised July 27, 2001; accepted July 27, 2001.

This work was supported by the Japan Science and Technology Corporation (JST) through Precursory Research for Embryonic Science and Technology (PRESTO) awarded to H.N., the Science Promotion Foundation of Gifu University School of Medicine (H.N.), the Ministry of Education, Culture, Sports, Science and Technology of Japan (Grants-in-Aid for Scientific Research 12680729 to H.N. and 12710038 to M.W.), and the Cooperative Research Program of Primate Research Institute, Kyoto University. We thank Dr. Susan Hockfield for her gift of the Cat-301 antibody.

Correspondence should be addressed to Hiroyuki Nakamura, Department of Anatomy, Gifu University School of Medicine, Gifu 500-8705, Japan. E-mail: hiron@cc.gifu-u.ac.jp.

Copyright (C) 2001 Society for Neuroscience $\quad 0270-6474 / 01 / 218174-14 \$ 15.00 / 0$
}

part of area 5 (5ip) receive visual inputs from PO and send visual information and motor-related commands to dorsal premotor area 6 (Petrides and Pandya, 1984; Kurata, 1991; Tanne et al., 1995; Johnson et al., 1996; Matelli et al., 1998; Shipp et al., 1998; Caminiti et al., 1999). On the other hand, the neurons related to the hand-grasping movements are recorded in parietal areas 5 and 7, including MIP, LIP, and the anterior intraparietal area (AIP), in addition to the frontal area ventral premotor area 6 , namely area $6 \mathrm{a} \beta$ of Vogt and Vogt (1919), area FCBm of von Bonin and Bailey (1947), or area F5 of Matelli et al. (1985) (Mountcastle et al., 1975; Rizzolatti et al., 1988, 1990; Taira et al., 1990; Gallese et al., 1994; Sakata and Taira, 1994; Jeannerod et al., 1995; Sakata et al., 1995; Murata et al., 1996, 1997, 2000). AIP projects to ventral premotor area 6 (Petrides and Pandya, 1984; Matelli et al., 1986; Johnson et al., 1996); however, the visual inputs to AIP are not known. Because the 3-D visual information is processed in LIP (Gallese et al., 1994; Gnadt and Mays, 1995; Shikata et al., 1996; Endo et al., 1998; Gnadt and Beyer, 1998; Gnadt, 2000), we hypothesize that the 3-D information should be sent from LIP to AIP.

In this study, we investigated the connections that may underlie the transformation of 3-D vision to prehensile hand movements in the lateral bank of the intraparietal sulcus of the macaque monkey. A bidirectional tracer, wheat germ agglutinin conjugated to horseradish peroxidase (WGA-HRP), was first injected into the posterior part of LIP under the control of unit recording for 3-D visual stimuli. Several orthograde and retrograde tracers were then injected into V3A and AIP to show the overlap of the 
Left

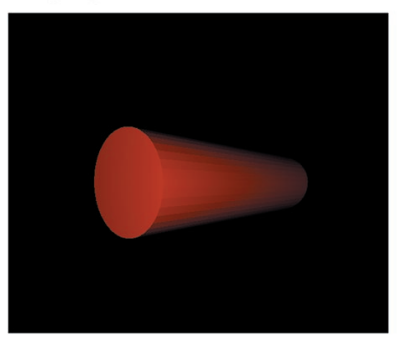
Right

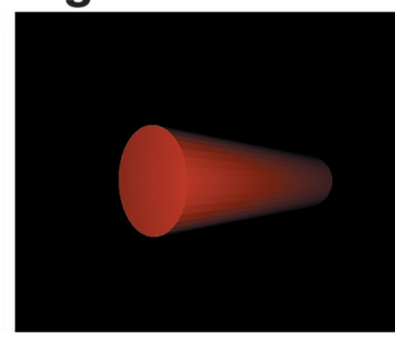

Merged

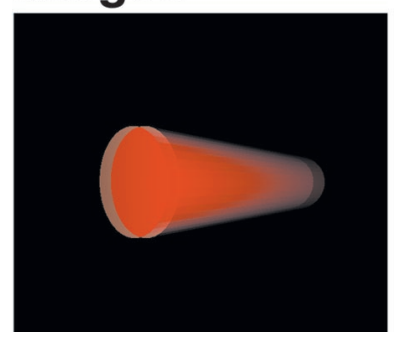

Figure 1. One of the three-dimensional visual stimuli used in this study. Left and Right show the stereoscopic stimuli for left and right eyes presented in turn with a shuttered polarizing filter $(60 \mathrm{~Hz}$ for each eye) and seen through polarizing glasses. Merged shows the same stimulus on the monitor seen without the glasses.

terminals projecting from $\mathrm{V} 3 \mathrm{~A}$ and the neurons projecting to AIP in the posterior part of LIP.

\section{MATERIALS AND METHODS}

The experiments were performed with 10 adult macaque monkeys (Japanese monkey, Macaca fuscata) of both genders weighing $5.6-9.5 \mathrm{~kg}$. All animals were cared for in accordance with the National Institutes of Health Guide for the Care and Use of Laboratory Animals and the Guide for the Care and Use of the Laboratory Primates of the Primate Research Institute of Kyoto University.

Electrophysiological recordings. The neurons that responded to 3-D visual stimuli were recorded from the cortex lining the posterior part of the intraparietal sulcus of four monkeys. The animals were sedated with an intramuscular injection of ketamine hydrochloride $(10 \mathrm{mg} / \mathrm{kg})$ and were anesthetized with an intravenous injection of sodium pentobarbital $(30 \mathrm{mg} / \mathrm{kg})$. Supplemental doses of sodium pentobarbital $(8 \mathrm{mg} / \mathrm{kg})$ were given as needed to maintain a surgical level of anesthesia. Scleral search coils were implanted in both eyes, and a head fixation ring was attached to the skull. Recording chambers (inner diameter, $20 \mathrm{~mm}$ ) were placed on the posterior part of the intraparietal sulcus of both hemispheres. After recovering from surgery, the animals underwent fluid restriction for $5 \mathrm{~d}$ a week and only received fluid as a reward for the neurophysiological recordings; the other $2 \mathrm{~d}$ during the weekend, they were allowed ad libitum access to water. Food consumption was not limited at all. The animals sat in a primate chair facing a back-projection display (TPP1670 HDB Super Projector, Ikegami, Tokyo; $157 \mathrm{~cm}$ width by $87 \mathrm{~cm}$ height; $1280 \times 984$ pixels). To receive their fluid reward, the animals were required to fixate their gaze on a small spot of light located at the center of the computer display during the 3-D visual stimuli presentation. One second after the fixation spot appeared, the 3-D visual stimuli were presented for $1200 \mathrm{msec}$. After the visual stimuli disappeared, the animals were required to continue fixation for $800 \mathrm{msec}$ more and were then given a liquid reward. Recordings were made in six hemispheres of four monkeys with tungsten electrodes (FHC, Brunswick, ME) from a $15 \mathrm{~mm}$ diameter area of cortex within the recording chamber placed over the posterior parietal sulcus. The 3-D, stereo visual stimuli (Fig. 1, Left, Right) were generated with a horizontal disparity difference for the right and left eyes on a workstation (Indigo2, Silicon Graphics, Mountain View, CA) using a in-house software package (Solidray, Yokohama, Japan). The binocular stimuli $(1280 \times 492$ pixels for each eye $)$ were presented alternatively on the back-projection display with an electronically shuttered polarizing filter (Projection Z Screen, StereoGraphics,
San Rafael, CA; refresh rate, $60 \mathrm{~Hz}$ for each eye). The alert, behaving monkeys rested on a chair at $57 \mathrm{~cm}$ in front of the display with a pair of opposed polarizing lenses that matched the two states of the liquid crystal-modulated polarizing filter placed directly in front of the monkey's eyes. The $3-\mathrm{D}$ stimuli used were $20-60^{\circ}$ in size and of various orientations. Several simple 3-D objects, such as bar, cylinder, pyramid, cone, square plate, and disk, were presented with four different orientations of $45^{\circ}$ difference in three different planes (i.e., horizontal, vertical, and frontal planes). For the objects with an optimal response, changes such as width, length, and thickness were examined with double-step size differences. The neuronal activity to the most effective object was again recorded with binocular disparity using the polarizing glasses (Fig. 1, Left, Right) and without disparity cue without the glasses (Fig. 1, Merged). When the neuron was more active during the recording with the polarizing glasses than during the recording without the glasses, we consider the neuron responsive to the 3 -D objects. Each recording session was $\sim 5$ $\mathrm{hr}$ or longer each day, and the recording periods were from 3 to 6 months per hemisphere. After the recordings were finished, microelectrode penetrations were performed at known coordinates, and electrolytic lesions were made to locate them after histology.

$W G A-H R P$ injections into the LIP. After the recordings, we placed a recording $1 \mu \mathrm{l}$ Hamilton syringe (Crist Instruments, Damascus, MD) through the recording chamber to one of the most typical recording sites. We first recorded the unit responses to the 3-D visual stimuli through the syringe and then injected $0.05 \mu \mathrm{l}$ of $5 \%$ solution of WGA-HRP (Toyobo, Osaka, Japan) dissolved in physiological saline into LIP. After $48 \mathrm{hr}$, the animal was anesthetized with a lethal dose of sodium pentobarbital (80 $\mathrm{mg} / \mathrm{kg}$, i.v.) and perfused through the ascending aorta with 21 of heparinized saline followed by 41 of $4 \%$ paraformaldehyde in $0.1 \mathrm{M}$ sodium phosphate buffer (PB), $\mathrm{pH}$ 7.4. Frontal sections, $50 \mu \mathrm{m}$ thick, were cut on a freezing microtome, and one-in-five serial sections were reacted with tetramethyl benzidine to visualize WGA-HRP labeling (Mesulum, 1978).

Analysis of the recording sites. To find out from which part of the cortex we recorded the 3-D visual responses, 1-in-10 serial sections were mounted on gelatinized glass slides and stained with cresyl violet for the Nissl substance. Another 1-in-10 serial sections were stained for myeloarchitecture according to the Gallyas method (1979) to evaluate areas LIPd, LIPv, VIP, 7a, and V3/V3A, because previous studies had used myelin staining to help define the borders of the areas (Seltzer and Pandya, 1980; Van Essen et al., 1986; Felleman and Van Essen, 1987; Gattass et al., 1988; Andersen et al., 1990a; Blatt et al., 1990; Colby et al., 1993a; Felleman et al., 1997; Beck and Kaas, 1999). The needle tracks were examined using a microscope (Axiophoto, Zeiss, Jena, Germany) equipped with a Pentium PC-controlled 3-D motorized stage, and the locations of recording sites were digitized with a 3-D microscopic data analyzing system (Neurolucida, MicroBrightField, Colchester, VT). The $x-y$ parameters of the sections were monitored with a motorized stage, and the recording sites were plotted with a mouse pointer on the monitor view projected through a camera lucida in the microscopic objective view. The areal borders were added to the drawing using adjacent myelinstained sections. To analyze the recording sites, the orientation index was calculated using the electrophysiological data and was defined as $(1-$ the response to the stimulus in the optimal orientation in 3-D space/the response to the stimulus in the orthogonal orientation).

Tracer injections into V3A and AIP. In one alert monkey, we first electrophysiologically recorded the neuronal responses from V3A and the areas around it to evaluate the location of V3A, and then injected tracers into V3A of both hemispheres. To identify the location and extent of V3A, we examined the distribution of gaze-dependent visual neurons with a single-unit recording for visual responses, because visual activity of neurons in V3A has been reported to be modulated by gaze (Galletti and Battaglini, 1989; Nakamura and Colby, 2000). The electrophysiological recording was prepared as described above. The monkey was then trained to fixate a dim light on the monitor as described above, and the receptive field was determined by moving a small slit or spot of light. The fixation point was located on the monitor at nine different points (i.e., top, middle, and bottom levels and right, center, and left for each level) with a $20^{\circ}$ difference, and the visual stimulus was centered on the receptive field. The sizes of receptive fields were similar in V3 and $\mathrm{V} 3 \mathrm{~A}$, but the activity of the neurons in V3 did not depend on the direction of gaze. After recording for $>3$ months from both hemispheres, we placed a recording $1 \mu \mathrm{l}$ Hamilton syringe into the V3A under the guide of the unit recording and injected $0.05 \mu \mathrm{l}$ of 5\% WGA-HRP and 2 $\mu \mathrm{l}$ of $10 \%$ biotinylated dextran amine (BDA) (BDA-10,000, Molecular 

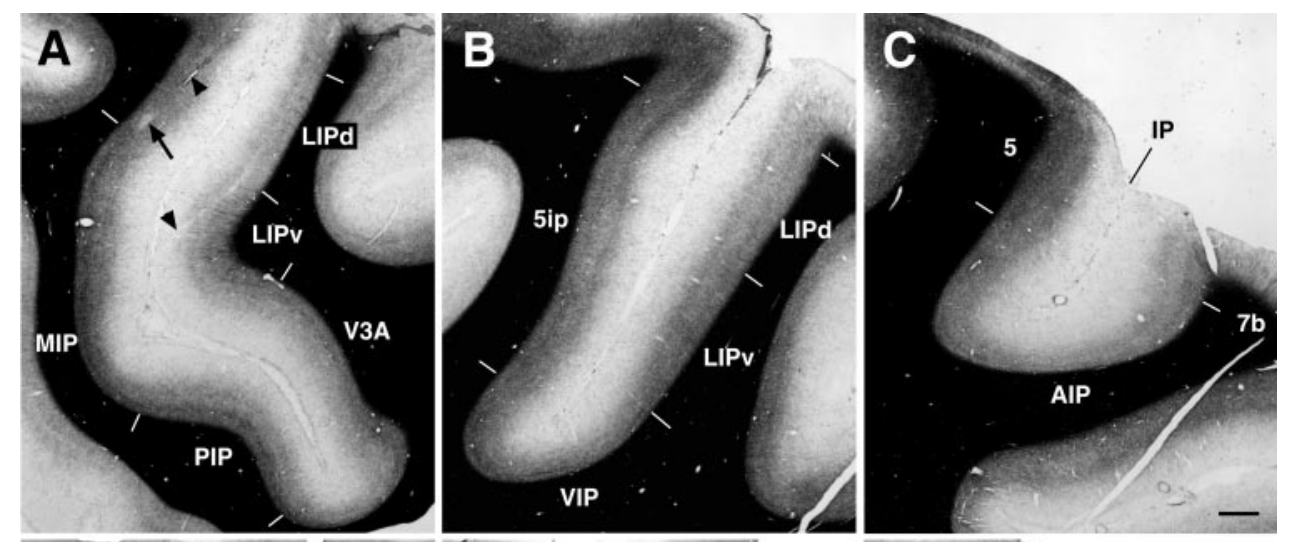

Figure 2. Photographs showing the areal differences of myeloarchitecture $(A-C)$ and Cat-301 immunostaining $(D-F)$ in the intraparietal areas. The sections of the top and bottom rows ( $A$ and $D, B$ and $E$, and $C$ and $F$ ) are at similar rostrocaudal levels. $A-C$ are from one of the monkeys in which we made electrophysiological recordings from the lateral intraparietal area $(L I P)$, and sections $D-F$ are from one of the monkeys in which we injected tracers into the anterior intraparietal area $(A I P)$ and visual area V3A. Arrowheads show the tracks of recording electrodes, and an arrow shows a recording site. Note that the layers of $A I P$ are thicker than those of $L I P$ and $V 3 A$ in the coronal sections. 5, Area 5; 5ip, intraparietal part of area 5 [part of PEa excluding the medial intraparietal area $(M I P)$ and $A I P]$; $7 b$, area $7 \mathrm{~b} ; I P$, intraparietal sulcus; $L I P d$, dorsal part of the lateral intraparietal area; $L I P v$, ventral part of the lateral intraparietal area; $P I P$, posterior intraparietal area; VIP, ventral intraparietal area. Scale bars, $1 \mathrm{~mm}$.
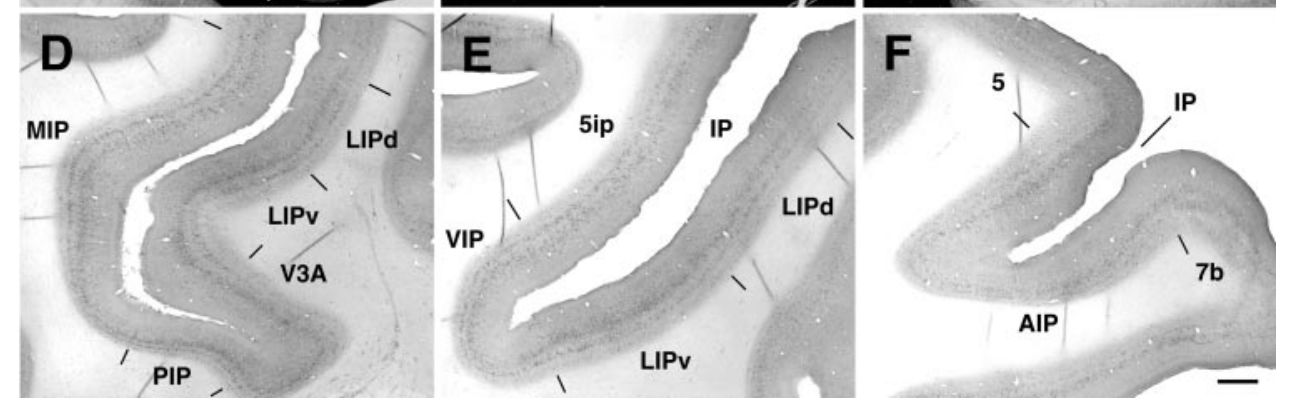

Probes, Eugene, OR). Because the injections were only placed in V3A without any injections into AIP, the data were not used in this study and will appear in another paper.

In the other five monkeys, we used magnetic resonance imaging (MRI) of the monkeys' brain map to obtain stereotaxic coordinates. We adapted the V3A borders evaluated above to the MRI images, and stereotaxically injected several tracers into both V3A and AIP of both hemispheres. From 2 to $10 \mathrm{~d}$ before the injection of tracers, the monkeys were induced to anesthesia with an intramuscular injection of ketamine hydrochloride $(10 \mathrm{mg} / \mathrm{kg})$ with atropine sulfate $(0.1 \mathrm{mg} / \mathrm{kg})$ to reduce respiratory secretions and anesthetized with an intravenous injection of sodium pentobarbital $(30 \mathrm{mg} / \mathrm{kg})$. A supplemental dose of sodium pentobarbital $(8 \mathrm{mg} / \mathrm{kg})$ was injected intravenously as needed to maintain the anesthesia. One millimeter interval slices of $1 \mathrm{~mm}$ thickness 3-D gradient echo images were taken using 0.2 T MRI (Profile, General Electronic, Milwaukee, WI)

All tracer injection procedures were conducted under aseptic conditions. We first injected tracers of long survival periods into the left V3A and AIP, and $10 \mathrm{~d}$ after the first injections, we then injected tracers of short survival periods into the right V3A and AIP. The animals were sedated with an intramuscular injection of ketamine hydrochloride (10 $\mathrm{mg} / \mathrm{kg}$ ) and anesthetized with an intravenous injection of sodium pentobarbital $(30 \mathrm{mg} / \mathrm{kg})$. Supplemental doses of sodium pentobarbital (8 $\mathrm{mg} / \mathrm{kg}$ ) were given as needed to maintain a surgical level of anesthesia. The skin of the head was midline incised, and a craniotomy was made over the left intraparietal sulcus. Up to three tracers were pressure injected using 1 and $5 \mu$ l Hamilton syringes (Hamilton, Reno, NV) or glass micropipettes with a Picospritzer 2 (General Valve, Fairfield, NJ). In the left hemisphere, $1 \mu \mathrm{l}$ of $10-20 \%$ BDA (BDA-3,000, Molecular Probes) was injected into V3A, and 1-3 $\mu$ l of $2 \%$ Fast blue (FB) (Sigma, St. Louis, MO) was injected into AIP. In one of the monkeys, 1-3 $\mu$ l of $10 \%$ Nuclear Yellow (NY) (Sigma) was also injected in the left AIP; in this animal, tracers were not injected into the right AIP. Ten days after the first injections, the monkeys were anesthetized again as described above, and a craniotomy was made over the right intraparietal sulcus. Up to three additional injections were placed into V3A and AIP contralateral to the first injections. We injected $0.03-0.05 \mu \mathrm{l}$ of 5\% WGA-HRP and $1 \mu \mathrm{l}$ of $10 \%$ tetramethylrhodamine dextran (Rd) (Molecular Probes) into V3A and $1-3 \mu \mathrm{l}$ of $10 \% \mathrm{NY}$ into AIP using 1 and $5 \mu \mathrm{l}$ Hamilton syringes or glass micropipettes with the Picospritzer 2. At $48 \mathrm{hr}$ after the second surgery, the animal was anesthetized with a lethal dose of sodium pentobarbital $(80 \mathrm{mg} / \mathrm{kg}$, i.v. $)$ and perfused through the ascending aorta with 21 of heparinized saline followed by 41 of $4 \%$ paraformaldehyde in PB.
After $2 \mathrm{~d}$ immersion of the brains in the PB with $20 \%$ glycerol, $50-\mu \mathrm{m}$-thick sections were cut frozen in the frontal plane and collected in PB. One-in-five serial sections were mounted on gelatinized slides from $0.45 \% \mathrm{NaCl}$ solution and observed under a fluorescent microscope (Axiophoto, Zeiss). The filters used were as follows: for Rd, excitation 534-558 nm, emission $580 \mathrm{~nm}$; for FB and NY, excitation 390-420 nm, emission $425 \mathrm{~nm}$. Photographs of these sections were taken, and images were merged using a fluorescent microscope (Axioplan 2, Zeiss) and a CCD camera (AxioCam, Zeiss) equipped with a Pentium PC and graphic software (AxioVision 2.05, Zeiss). Another one-in-five serial section was processed for WGA-HRP visualization. For BDA histochemistry, another one-in-five serial section was processed with the Elite $\mathrm{ABC}$ kit (Vector, Burlingame, $\mathrm{CA}$ ) and diaminobenzidine tetrahydrochloride (Wako) with glucose oxidase (Sigma) (Shu et al., 1988). These sections were observed and photographed under a bright-field and polarizing microscope (Axiophoto, Zeiss) equipped with a Pentium PCcontrolled 3-D motorized stage. The contours of the sections, borders between the cortex and the white matter, and locations of the labeled neurons and terminals were digitized with a 3-D microscopic data analyzing system (Neurolucida, MicroBrightField).

Myelin staining and Cat-301 immunostaining. Because previous studies had used myelin staining to help define the borders of intraparietal areas (Seltzer and Pandya, 1980; Van Essen et al., 1986; Felleman and Van Essen, 1987; Gattass et al., 1988; Andersen et al., 1990a; Blatt et al., 1990; Colby et al., 1993a; Felleman et al., 1997; Beck and Kaas, 1999), 1-in-10 serial sections of all the animals were processed for the physiological development to visualize myeloarchitecture (Gallyas, 1979) to determine areal borders. In the V3A and AIP injection cases, other 1-in-10 sections were reacted immunocytochemically with Cat-301 antibody (Hockfield et al., 1983; Hendry et al., 1984, 1988), because previous studies had found it a useful marker for the magnocellular pathway and parietal areas (Hendry et al., 1984, 1988; McGuire et al., 1989; DeYoe et al., 1990). The antibody was kindly provided by Dr. Susan Hockfield (Yale University School of Medicine, New Haven, CT). We also stained another two 1-in-10 sections for cytochrome oxidase histochemistry (Carroll and Wong-Riley, 1984) and SMI-32 immunocytochemistry (Hof and Morrison, 1995); however, these sections were not very helpful in finding the borders of the intraparietal sulcul areas. The myelin and Cat-301 stained sections were used to visualize the borders of intraparietal cortical areas and thus confirm that injection sites intended for particular areas (AIP, $\mathrm{V} 3 \mathrm{~A}$ ) indeed had been in those areas.

The nomenclature used for the cortical areas correlated with that used in previous studies (Andersen et al., 1990a; Blatt et al., 1990). We used $5 \mathrm{ip}$ for the area situated in the middle of the medial bank of the 


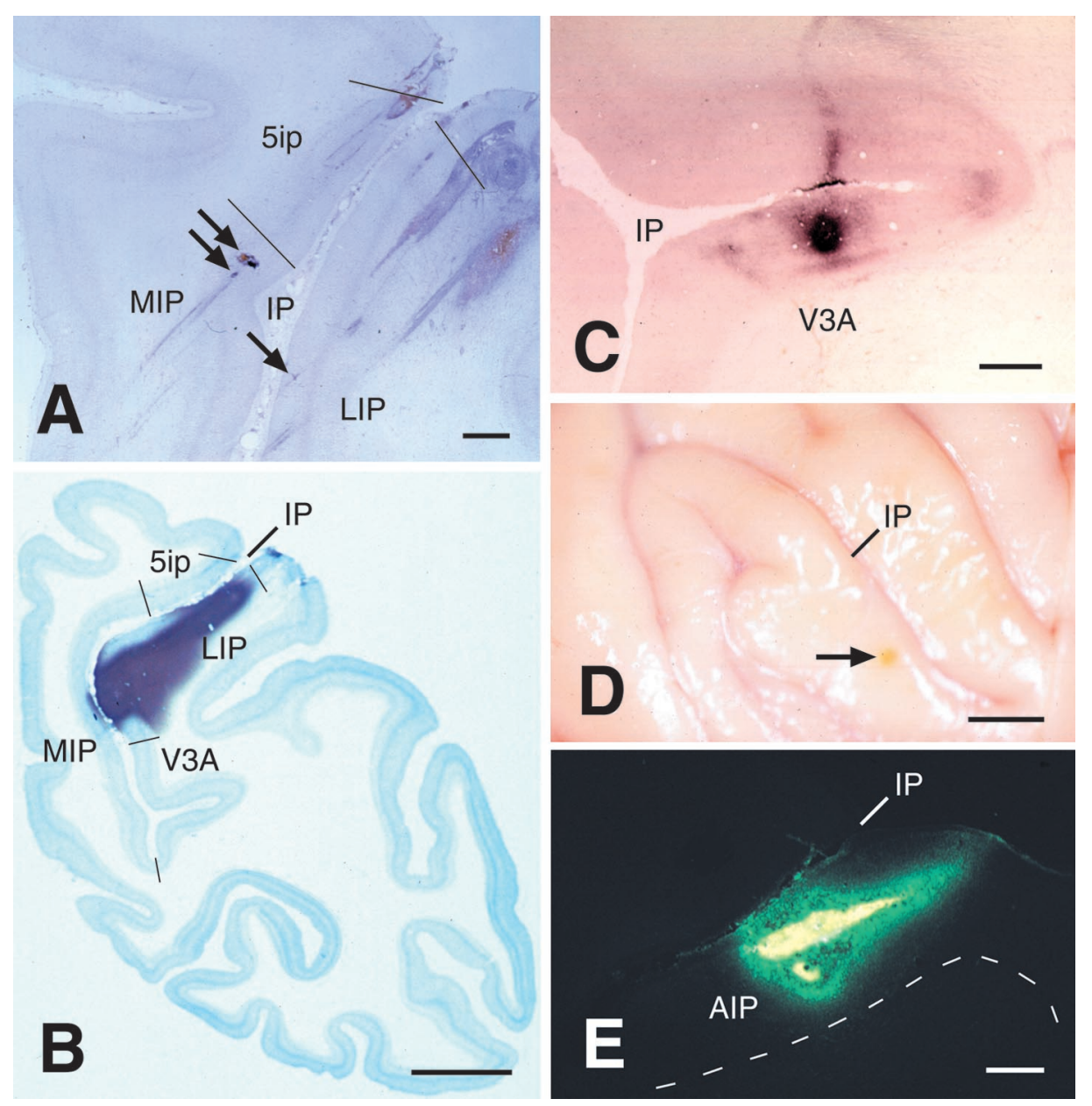

Figure 3. A, Nissl staining showing the gliosis along the recording needle tracks and the spot of gliosis at the recording sites (arrows). An arrow (bottom right) is in the lateral intraparietal area $(L I P)$, and two arrows (middle) are in the medial intraparietal area $(M I P) . B$, Injection site of WGA-HRP at one of the recording sites in LIP. C, Injection site of BDA in area $V 3 A$. $D$, Surface of the cortex showing back-diffusion of the injected Fast blue through the needle tract (yellow spot at the arrow) after the injection of the tracer into the anterior intraparietal area $(A I P) . E$, Injection site of Nuclear Yellow into AIP. The dashed line shows the border between the gray and white matter. $C-E$ are flipped horizontally, so they appear on the right side of the brain. 5ip, Intraparietal part of area 5 [part of PEa excluding the medial intraparietal area $(M I P)$ and $A I P] ; I P$, intraparietal sulcus. Scale bars: $A, C, E, 1 \mathrm{~mm} ; B, D, 5 \mathrm{~mm}$. intraparietal sulcus, i.e., part of the area PEa (Pandya and Seltzer, 1982) excluding the MIP and the cortex in the rostral part of the sulcus facing the AIP.

\section{RESULTS}

\section{Myeloarchitecture and Cat-301 staining of the intraparietal areas}

Figure 2 shows the myelin-stained and Cat-301-immunostained coronal sections to reveal areal borders of the intraparietal cortex. Where the sections were stained clearly, it was easy to recognize the borders of different areas. The tracks of the electrode penetrations (Fig. 2A, arrowheads) and the recording sites (Fig. $2 A$, arrow) were also apparent in the myelin sections as in the Nissl sections.

In the myelin-stained sections, densely stained bilaminar layers of the inner and outer bands of Baillarger were apparent in LIP (Fig. 2B). The bands of Baillarger were stained more densely in the ventral (LIPv) than in the dorsal (LIPd) part of the LIP. The densely stained bilaminar layers were also apparent in the posterior, curved portion of LIP (Fig. $2 A$ ). Densely myelinated, thick fibers ran perpendicular to the layers of LIP. In V3A, the outer band of Baillarger was wider but less densely stained than in the LIP, and the inner band of Baillarger was a thin layer that was densely stained and appeared as a clear band. In the posterior intraparietal area (PIP), the area located posterior to VIP, medial to V3 and V3A, and lateral to MIP and PO (Colby et al., 1988), densely stained bilaminar bands were observed, and the inner band of Baillarger was more densely stained than the outer band. In layer 3, many medium-sized myelinated fibers ran parallel to the layer, and in layer 1, thicker myelinated fibers were seen running parallel to the layer. In MIP, the inner band of Baillarger was thin and densely stained, whereas the outer band was not clear but seen as a densely stained wide layer. In the deep part of layer 3, layer 4, and layer 5, medium-sized, densely stained fibers ran parallel to the layers. In the middle to superficial layer 3 , fine fibers were seen running in both parallel and perpendicular directions to the layer. In the ventral intraparietal area (VIP), the inner band of Baillarger was very thin but densely stained, whereas the outer band of Baillarger was not clearly seen (Fig. $2 B)$. In layer 3 , thick myelinated fibers ran parallel to the layer. In area 5ip, the bilaminar bands of Baillarger were densely stained but were not so apparent as in LIP. Area 5 on the crown of the postcentral gyrus was very densely stained, and thick myelinated fibers were seen to run from layer 6 to the upper part of layer 3 . In AIP, the bilaminar bands of Baillarger were wider but not as densely stained as in LIP. The bilaminar pattern was thus not as apparent in AIP as in LIP. In most cases, the myelinated fibers in AIP were not stained as densely as those in LIP, but in some cases, they were stained as densely as those in LIPv. The staining difference in the dorsal and ventral parts, which was apparent in LIP, was not observed in AIP. The myelinated fibers were finer in AIP than in LIP, and a dense mesh of fine myelinated fibers was seen throughout the layers of AIP.

The Cat-301-immunostained sections were also useful in determining the borders between the parietal areas. In the LIP, 
immunoreactive neurons were distributed in three bands located in layers 3, 5 and 6 (Fig. 2E). Large pyramidal neurons and medium to large-sized nonpyramidal neurons were stained in the lower part of layer 3 and in layer 5. Several small to medium-sized pyramidal and nonpyramidal neurons were also Cat-301 positive in the upper part of layer 3 and in layer 6. The staining pattern was similar in both LIPd and LIPv, although fewer Cat-301positive neurons were observed in LIPd than in LIPv. Large cells were more abundant in LIPv than in LIPd, and the laminar structure was more apparent in LIPv than in LIPd. The same histological appearance was maintained in the posterior curved part of the LIP (Fig. 2D). In V3A, the three-layered pattern was also apparent, but the size of the Cat-301-positive neurons was small. In V3, the Cat-301-positive neurons were scattered throughout the width of layer 3. The Cat-301-positive neurons were also distributed widely in layers 5 and 6 . As a result, the three-layered pattern in V3 was not as apparent as in LIP, and it appeared as if the Cat-301-positive neurons were distributed over layers 3-6 with a thin lamina of cell-free zone in layer 4. In PIP, the laminar structure was not so apparent, but large pyramidal neurons in layers 3 and 5 were strongly Cat-301 positive. In MIP, Cat-301-positive neurons were more compactly arranged in the deep layer 3 than in PIP, and some large pyramidal and nonpyramidal Cat-301-positive neurons were scattered in layer 5. Accordingly, they showed as distinct a laminar structure as that seen in LIPv. Compared with LIPv, however, fewer Cat-301-positive neurons were localized in the upper part of layer 3 and in layer 6 of MIP. In VIP, the number of Cat-301-positive neurons was smaller than in LIP or MIP, but these neurons were compactly arranged and the lamination was distinct. In area 5ip, a smaller number of Cat-301-positive neurons was observed, and the neurons showed a less distinct laminar pattern compared with MIP. In area 5, no lamination was found, and only a few Cat-301positive neurons were scattered in layer 3. In AIP, Cat-301positive neurons were found only in the deeper half of layer 3 , and no large neurons were Cat-301 positive in layers 5 and 6 .

\section{Electrophysiological recordings from LIP}

Because our previous study showed that neuronal responses to 3-D visual objects were recorded in LIP but not in V3A (Shikata et al., 1996), we mapped the recording sites for 3-D visual objects (Figs. $3 A, 4 A$ ). To clarify whether the neurons responded to a 3-D visual object but not to the orientation of an object in the two-dimensional monitor screen, we examined the orientation tuning of the neuronal response to the object in the 3-D space. The orientation tuning was examined by varying the surface or axis orientation of stimulus around the sagittal, horizontal, or vertical axis (sagittal axis was parallel to the direction of gaze; horizontal and vertical axes were parallel to the frontal plane). When the neurons were more responsive to the stimuli of one orientation than of the other orientations in 3-D space, we considered the neurons to be $3-\mathrm{D}$ orientation selective. The responses to the stimulus presented to either the left or right eye (Fig. 1, Left, Right) or to the 3-D stimulus viewed without polarizing glasses (Fig. 1, Merged) were much less than those to the same stimulus viewed binocularly with polarizing glasses. Thus, all these neurons showed a disparity effect, i.e., the response activity was stronger with polarizing glasses compared with the response to the same stimuli without the glasses. The response activities linearly increased as the size of the object increased to reach some limit, and then, when the size got bigger than the size

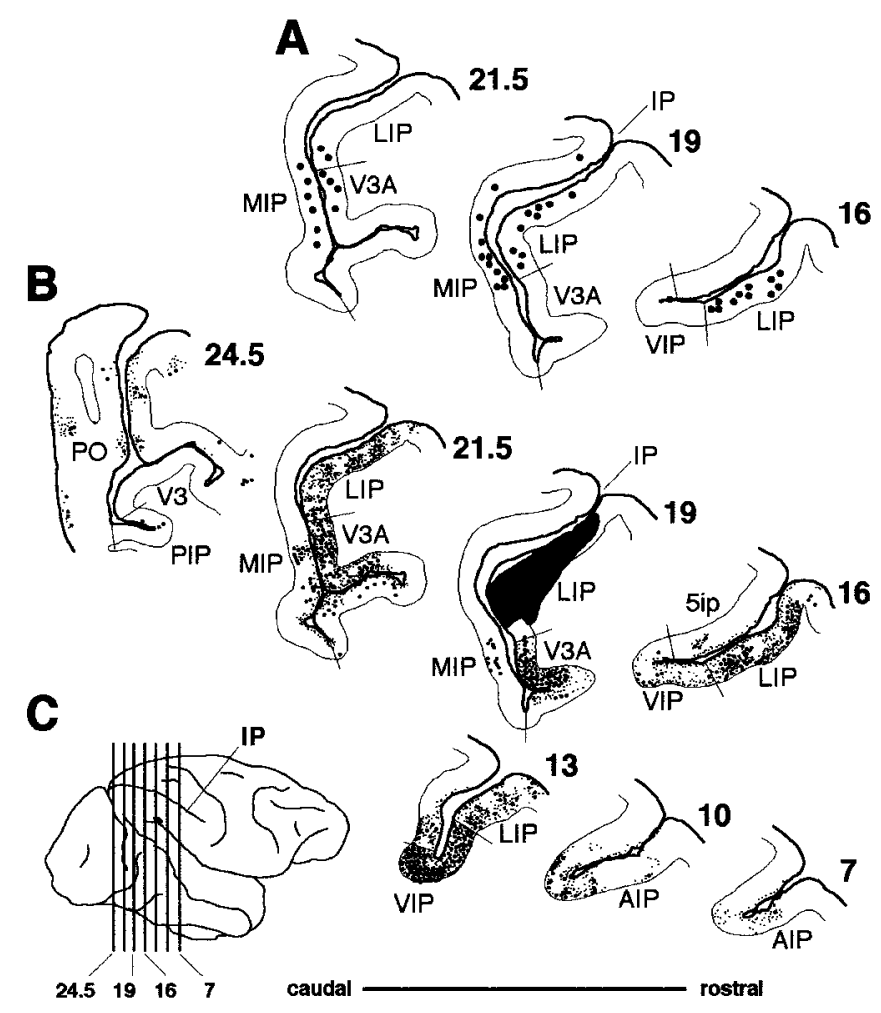

Figure 4. Recording sites of the neurons that responded to threedimensional visual stimuli and the distribution of labeling after injecting WGA-HRP into one of the recording sites in the posterior part of the lateral intraparietal area $(L I P) . A$, The recording sites of neurons with an orientation index of $>0.4$ (filled circles). $B$, Drawings showing orthogradely labeled terminals (dots) and retrogradely labeled neurons ( filled circles) after an injection of WGA-HRP ( filled area on section 19) into the three-dimensional visual responsive region of the posterior part of LIP. C, Drawing of the hemisphere showing the levels of the frontal sections in $A$ and $B$. The numerals indicate the distance in millimeters from the rostral tip of the intraparietal sulcus. 5ip, Intraparietal part of area 5 [part of $\mathrm{PEa}$ excluding the medial intraparietal area $(M I P)$ and $A I P] ; I P$, intraparietal sulcus; $P O$, parieto-occipital area; $P I P$, posterior intraparietal area; $V 3$, visual area V3; VIP, ventral intraparietal area.

limit, the response decreased, thus suggesting the existence of surround inhibitory "off" receptive field.

Our observations were biased toward visually responsive units, but we also observed neurons that were active during hand reaching. For example, in one of the monkeys in which 61 penetrations were made and 462 neurons were isolated, visual and visual-related neurons were 374 (81\%), including 23 neurons that fired to both visual stimuli and reaching hand movements $(5 \%)$. Six neurons were active during reaching hand movements but not during visual testing (1.3\%). A total of 38 neurons $(8.2 \%)$ responded to $3-\mathrm{D}$ visual stimuli.

A total of 470 penetrations were made in six hemispheres, and the responses of 73 visual neurons to 3-D stimuli were studied. On the Nissl-stained sections, the recording needle tracks were clearly seen as lines of gliosis (Fig. $3 A$ ). Some of the recording sites were apparent as the large spots of gliosis along the needle track (Fig. 3A, arrows), probably because of long recording sessions (usually $2-3 \mathrm{hr}$ for one recording site, sometimes $>5 \mathrm{hr}$ ). Of 73 neurons examined with 3 -D visual stimuli, 56 neurons showed statistically significant orientation preference. To be conservative, we chose 43 neurons with an orientation index of $>0.4$ to map the location of these neurons (Fig. $4 A$ ). For the 43 neurons that were 
activated with 3-D visual stimuli, of which 22 of that number were in LIP, 17 in MIP, and 4 in V3A. Seventeen neurons showed a preferred response to disk or square plate, whereas 26 preferred bar or cylinder. There neurons were intermingled, and we did not recognize any spatial segregation of these units. We recorded only in the posterior part of the intraparietal sulcus, so we did not record any units in the anterior part of LIP.

\section{Injection of WGA-HRP into LIP recording site}

After the recordings, we injected WGA-HRP into one of the most typical recording sites in the posterior part of LIP under the control of unit recording of neurons responding to the 3-D visual stimuli (Figs. 3B, 4B). The WGA-HRP injection covered most of the posterior part of LIP (Fig. $4 B$ ) and extended from layer 1 to layer 6. The injected WGA-HRP also diffused into the white matter below layer 6 , but because the injection track was limited to cortex and because WGA-HRP is not taken up by intact axonal shafts (Mesulum, 1982), we considered the resulting labeling to be the result of the cortical part of the injection. Orthogradely labeled terminals and retrogradely labeled neurons were found abundantly in LIP, VIP, and V3A and slightly in MIP, PIP, PO, and AIP. In AIP, WGA-HRP-labeled terminals were found mainly in layer 3, and a small amount was found in layer 4 (Fig. $5 A$ ). A few retrogradely labeled neurons were also found in layers 3 and 5. In V3A, many WGA-HRP-labeled neurons were found in layer 3, and only a few were found in layer 5 (Fig. 5B). WGA-HRP-labeled terminals were densely distributed in layer 1 , and a few were distributed in layers 3,5 , and 6 , but no labeling was seen in layer 4 of V3A. In PO, only a small number of WGAHRP-labeled neurons were observed in layer 3, and a few were observed in layer 5 (Fig. $5 C$ ). In addition, a few WGA-HRPlabeled terminals were found in layers 1 and 3. Other than V3A and $\mathrm{PO}$, we could not find any substantial projections from any of the other occipital visual areas after the LIP injection.

\section{Double tracer injections into V3A and AIP}

To confirm the connections from V3A to AIP relayed by LIP, we injected bidirectional tracers (WGA-HRP, BDA, and Rd) into V3A and retrograde tracers (FB and NY) into AIP. The injections into V3A and AIP were made without recording, but instead by reliance on MRI-derived stereotaxic coordinates, and were later confirmed as being in V3A and LIP by architectonics using myelin-stained sections and Cat-301-immunostained sections. Although we tried a total of 14 injections in V3A of 10 hemispheres, 2 were well localized within the V3A without any diffusion into the white matter or the other areas, and 5 were localized in the V3A with some diffusion into the white matter. For the latter cases, the injection needle track also got into the white matter except in one case. Although a total of 12 injections were made into AIP of nine hemispheres, 4 were well localized within AIP without any diffusion into area $7 \mathrm{~b}$ or the white matter, but 5 covered AIP and area $7 \mathrm{~b}$ with diffusion into the white matter. For the latter cases, the injection needle track was limited in the cortex in three cases and got into the white matter in two cases. We successfully injected both V3A and AIP in six hemispheres, and in five of them the injections were good enough to study the overlap of connections in LIP. Figure $3 C-E$ shows a few of these injection sites.

Figure 6 shows the distribution of labeling in one of the multiple tracer experiments. In this case, BDA was injected into V3A (Figs. $3 C, 6 A$ ), and FB (Figs. 3D, 6B) and NY (Fig. 6C) were injected into AIP far beyond the border between LIP and AIP.

The V3A injection of BDA was well confined to V3A in the lateral part of the annectant gyrus. The BDA injection was centered in the deep part of layer 3 and extended to the upper part of layer 3 and to layers 4 and 5. Because layers 3 and 5 are the main sources of corticocortical projection neurons in most cortical areas (Rockland and Pandya, 1979; Maunsell and Van Essen, 1983; Van Essen and Maunsell, 1983; Andersen et al., 1990a), the limited laminar extent of this injection probably did not result in any missed projections. In this case, orthogradely labeled terminals were mainly found in layer 4 , and some were found in layer 3 of the LIP (Fig. 6A). Retrogradely labeled neurons were found in layer 5 with a few in layer 3. Although most of the BDA labeling was distributed in the posterior part of LIP, BDAlabeled terminals were scattered throughout the rostrocaudal extent of LIP. Other than the labeling in LIP, only a few BDAlabeled fibers were found in layers 3, 4, and 6 of VIP and MIP.

The AIP injection of FB was placed in the lateral and posterior part of AIP and extended from layers 4 to 6 . Because layer 4 is the main target of forward corticocortical projection neurons in most cortical areas (Maunsell and Van Essen, 1983; Van Essen and Maunsell, 1983; Felleman and Van Essen, 1991), the limited laminar extent of this injection probably did not result in any missed projections from LIP to AIP. Although the injection spread into the white matter, the needle track did not, thus suggesting that no artifactual labeling resulted. It also included all the layers of the medial part of area $7 \mathrm{~b}$. Thus the results reflected the connections of AIP and area $7 \mathrm{~b}$. The connections of the
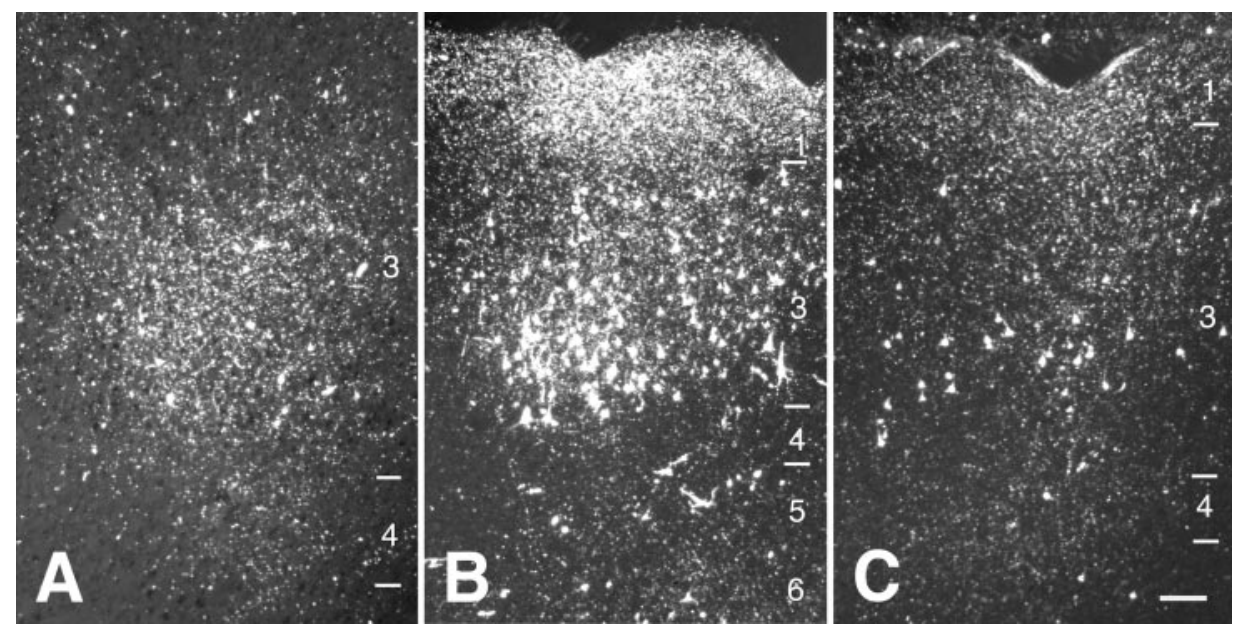

Figure 5. Dark-field photomicrographs showing orthogradely labeled terminals and retrogradely labeled neurons in the anterior intraparietal area (AIP) $(A)$, the visual area V3A $(B)$, and the parieto-occipital area $(\mathrm{PO})$ $(C)$ after the injection of WGA-HRP into a region of LIP in which three-dimensional responsive neurons were recorded. $A$, Labeled terminals in layers 3 and 4 of AIP. A few labeled neurons were also seen in layer 3. $B$, In V3A, many labeled neurons were seen in layer 3 , and only a few were observed in layer 5. Dense terminal-like labeling was seen in layer 1, and light terminal-like labeling was in layers 3 and 6 . $C$, Labeled neurons in layer 3 and labeled terminals mainly in layer 1 in the PO. Note that, as seen in $A$, the cortex in the coronal sections is thicker in AIP than in V3A or PO. Scale bar, $100 \mu \mathrm{m}$. 

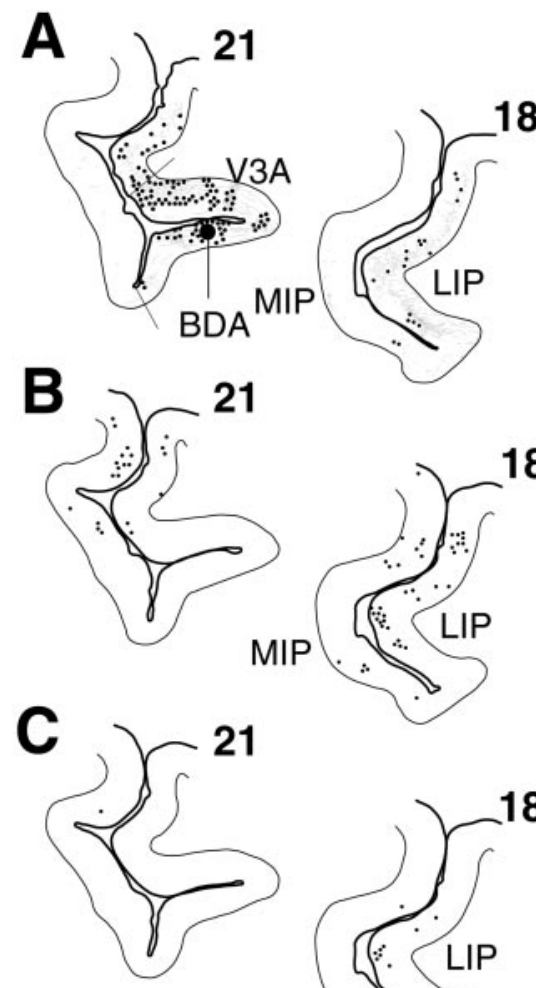

18

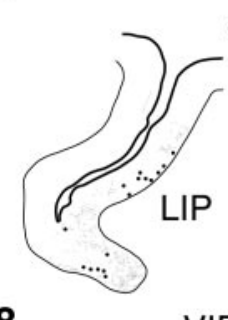

16
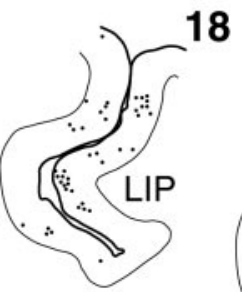

18
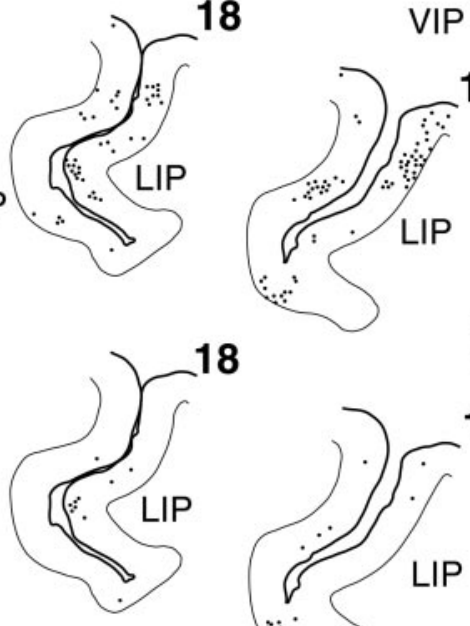

18

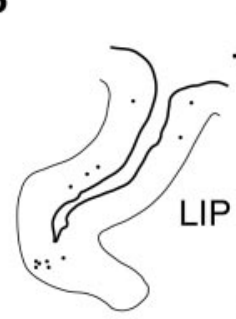

caudal

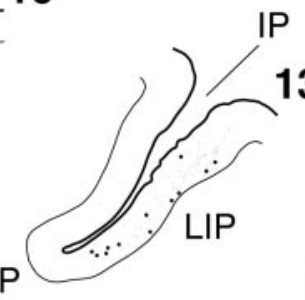

16

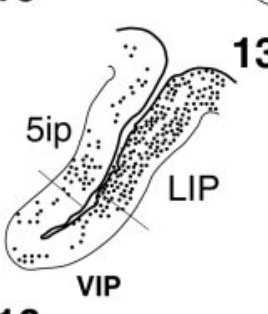

16

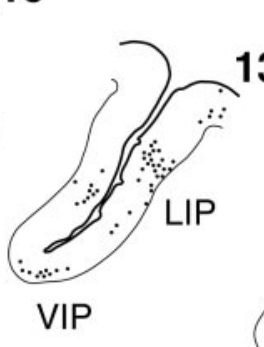

rostral

13

13
D
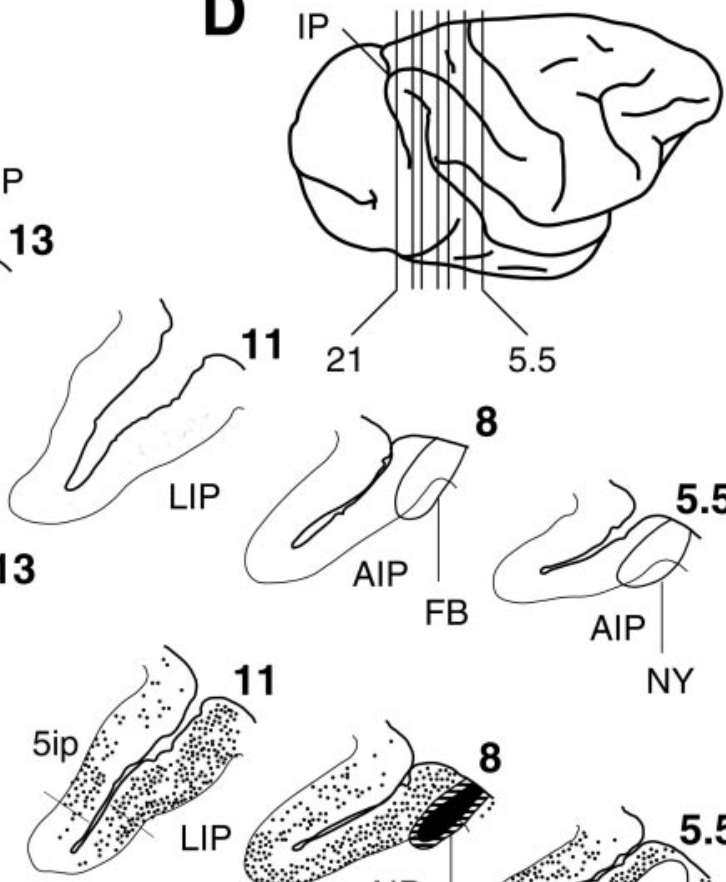

VIP

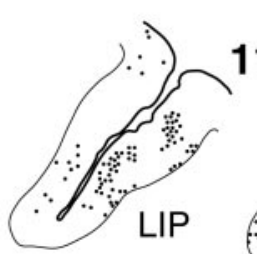

LIP

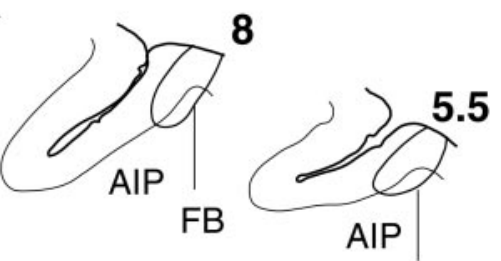

NY
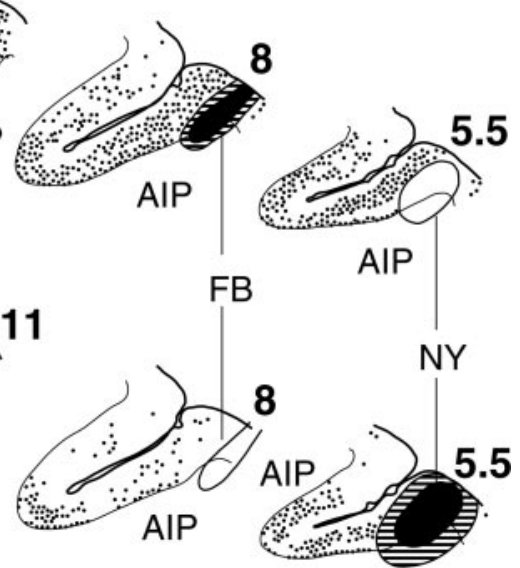

Figure 6. Distribution of labeling in the intraparietal areas demonstrating the overlap in the lateral intraparietal area $(L I P)$ of anterograde labeling from the visual area $V 3 A$ and retrograde labeling from the anterior intraparietal area $(A I P)$. Biotinylated dextran amine $(B D A)$ was injected into $V 3 A(A)$; Fast blue $(F B)$ and Nuclear Yellow $(N Y)$ were injected into $A I P(B, C)$. $A$, After the injection of $B D A$ into V3A, labeled axons with boutons or terminal arbors (lines) and neurons ( filled circles) were mainly distributed in LIP. B, After the injection of $F B$ into the posterior part of $A I P$, retrogradely labeled neurons ( filled circles) were distributed in $A I P, L I P$, the intraparietal part of area $5(5 i p)$, the ventral intraparietal area $(V I P)$, and the medial intraparietal area $(M I P) . C$, After the injection of $N Y$ into the anterior part of $A I P$, retrogradely labeled neurons ( filled circles) were distributed in $A I P, L I P$, 5ip, VIP, and MIP. D, Drawing of the hemisphere showing the levels of the frontal sections in $A-C$. The conventions are the same as those in Figure 4 .

intraparietal areas other than area 5ip observed in this case were similar to those found in the other cases in which the injection site was restricted in AIP. Retrogradely labeled neurons were found in AIP, LIP, 5ip, VIP, and MIP (Fig. 6B). Most of the labeling in area 5ip and part of the labeling in LIP were probably caused by the involvement of the injection site in area $7 \mathrm{~b}$. FB-labeled neurons were localized in layers 3 and 5 of AIP, VIP, and the anterior part of LIP. In LIP and MIP, FB-labeled neurons were localized mainly in layer 3. Because the injection of AIP included only layers $4-6$, it is possible that this injection did not fully label afferents to AIP. The main difference in the labeling of this case in comparison with other cases with large injections was a paucity of the labeled neurons in the VIP and the posterior part of the LIP.

The AIP injection of NY was placed in the anterior part of AIP $\sim 2 \mathrm{~mm}$ anterior of the FB injection. The NY injection extended from layers 3 to 6 and also covered the medial part of area $7 \mathrm{~b}$. Because the injection needle track got into the white matter, the results included the connections of AIP, area $7 \mathrm{~b}$, and the white matter below. As in the FB injection, the connections of the intraparietal areas except area 5 ip observed in this case were similar to those found in other cases in which the injection site was restricted in AIP. Retrogradely labeled neurons were distributed in AIP, LIP, 5ip, VIP, and MIP (Fig. 6C). The NY-labeled neurons were localized mainly in layer 3 of these areas. In LIP, retrogradely labeled neurons were more densely distributed in the anterior part, but some were also found in the posterior part. The orthograde fibers and terminals from V3A and retrogradely labeled neurons projecting to AIP thus overlapped not only in the posterior part of LIP but also throughout the rostrocaudal extent of LIP.

Figure 7 shows the data of another animal in which WGA-HRP was injected into V3A and NY was injected into AIP. The injection of WGA-HRP was localized in the lateral part of V3A covering layers 1-6 and the white matter above. The axons in the white matter were damaged by the injection needle, so uptake of WGA-HRP might have occurred and affected the results. However, because no projections to LIP from areas more posterior 


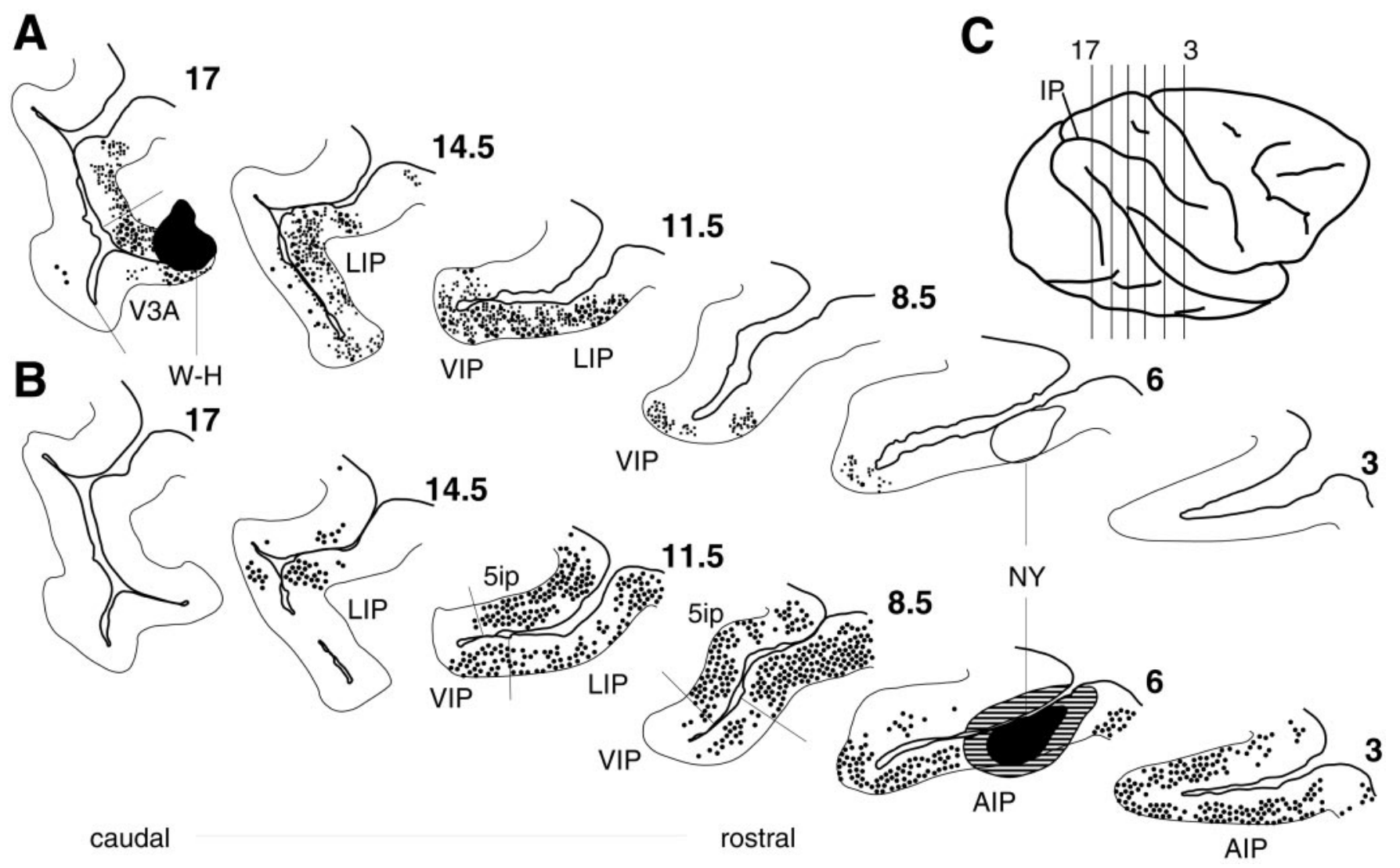

Figure 7. Distribution of labeling in the intraparietal areas after injection of WGA-HRP $(W-H)$ into the visual area $V 3 A$ and Nuclear Yellow $(N Y)$ into the anterior intraparietal area $(A I P)$. A, After the injection of WGA-HRP into the visual area V3A, labeled terminals (dots) and neurons ( filled circles) were distributed in the lateral intraparietal area $(L I P)$ and the ventral intraparietal area $(V I P)$. B, After the injection of $N Y$ into the posterior part of $A I P$, retrogradely labeled neurons ( filled circles) were distributed in $A I P, L I P$, the intraparietal part of area 5 (5ip), VIP, and the medial intraparietal area $(M I P)$. $C$, Drawing of the hemisphere showing the levels of the frontal sections in $A$ and $B$. The conventions are the same as those in Figure 4 .

than V3A have yet been reported, we considered the resulting labeling in LIP to be the result of the cortical part of the injection. Terminal-like labeling and labeled neurons were distributed in LIP, VIP, and MIP (Fig. 7A). In LIP, WGA-HRP-labeled terminals were found in layers 3 and 4 , and labeled neurons were found in layers 3 and 5. WGA-HRP labeling was distributed in a columnar manner in LIP. In VIP, terminal-like labeling was found in layers 1 and 3, and labeled neurons were found in layer 5. In MIP, only minimal terminal-like labeling was found in layer 1 , and a few labeled neurons were found in layer 3 .

The injection of NY was well confined to AIP and covered layers 1-6 but also diffused across the intraparietal sulcus into layers 1 to upper 3 in area 5ip. Although the injection spread into the white matter, the needle track did not, thus suggesting that no artifactual labeling resulted. The involvement of the anterior part of area 5ip may have labeled many neurons in the posterior part of area 5ip in this case. Retrogradely labeled neurons were distributed in layers 3 and 5 of AIP, LIP, 5ip, VIP, and MIP (Fig. $7 B)$. The terminals projecting from $\mathrm{V} 3 \mathrm{~A}$ and retrogradely labeled neurons projecting to AIP thus overlapped not only in the posterior part of LIP but also throughout the rostrocaudal extent of LIP in this case, too.

Figure 8 shows an overlap of the Rd-labeled fibers projecting from V3A and the distribution of NY- and FB-labeled neurons projecting to the ipsilateral and contralateral AIPs. Rd was injected into the lateral part of V3A covering layers 4-6 and diffused into the white matter. Because layers 3 and 5 are the main sources of corticocortical projection neurons in most cortical areas (Rockland and Pandya, 1979; Maunsell and Van Essen, 1983; Van Essen and Maunsell, 1983; Andersen et al., 1990a), the limited laminar extent of this injection probably resulted in a labeling of projections from layer 5 but not from layer 3. The injection needle passed through the white matter above layer 6 and thus damaged the axons. As a result, the uptake of Rd by passing fibers probably occurred and affected the results. Rdlabeled fibers were found in layers 3-6 of LIP, and Rd-labeled neurons were found mainly in layer 3 (Fig. $8 A$ ).

$\mathrm{NY}$ was injected into AIP covering layers 1-6. The injection also covered layers 1-2 of area 5ip across the intraparietal sulcus and diff used into layer 3 of area 5ip. Diff usion was also seen in areas $7 \mathrm{~b}$ and 5 . Although the injection spread into the white matter, the needle track did not, thus suggesting that no artifactual labeling resulted. The connections in the intraparietal areas, except area 5ip, observed in this case were similar to those found in the other cases in which the injection site was confined to AIP. Most of the labeling in area 5ip was probably caused by the involvement of the injection site in areas 5ip and $7 \mathrm{~b}$. Retrogradely labeled neurons were distributed in layers 3 and 5 of AIP, LIP, 5ip, VIP, and MIP (Fig. 8B). In LIP, NY-labeled neurons were localized in the rostrocaudal extent of the area. In the posterior part of LIP, the neurons and fibers labeled with Rd were found in the vicinity of AIP-projecting neurons labeled with NY (Fig. $9 A-C)$. Some of the NY-labeled AIP-projecting neurons were also found to project to V3A (Fig. $9 D-F$ ). 

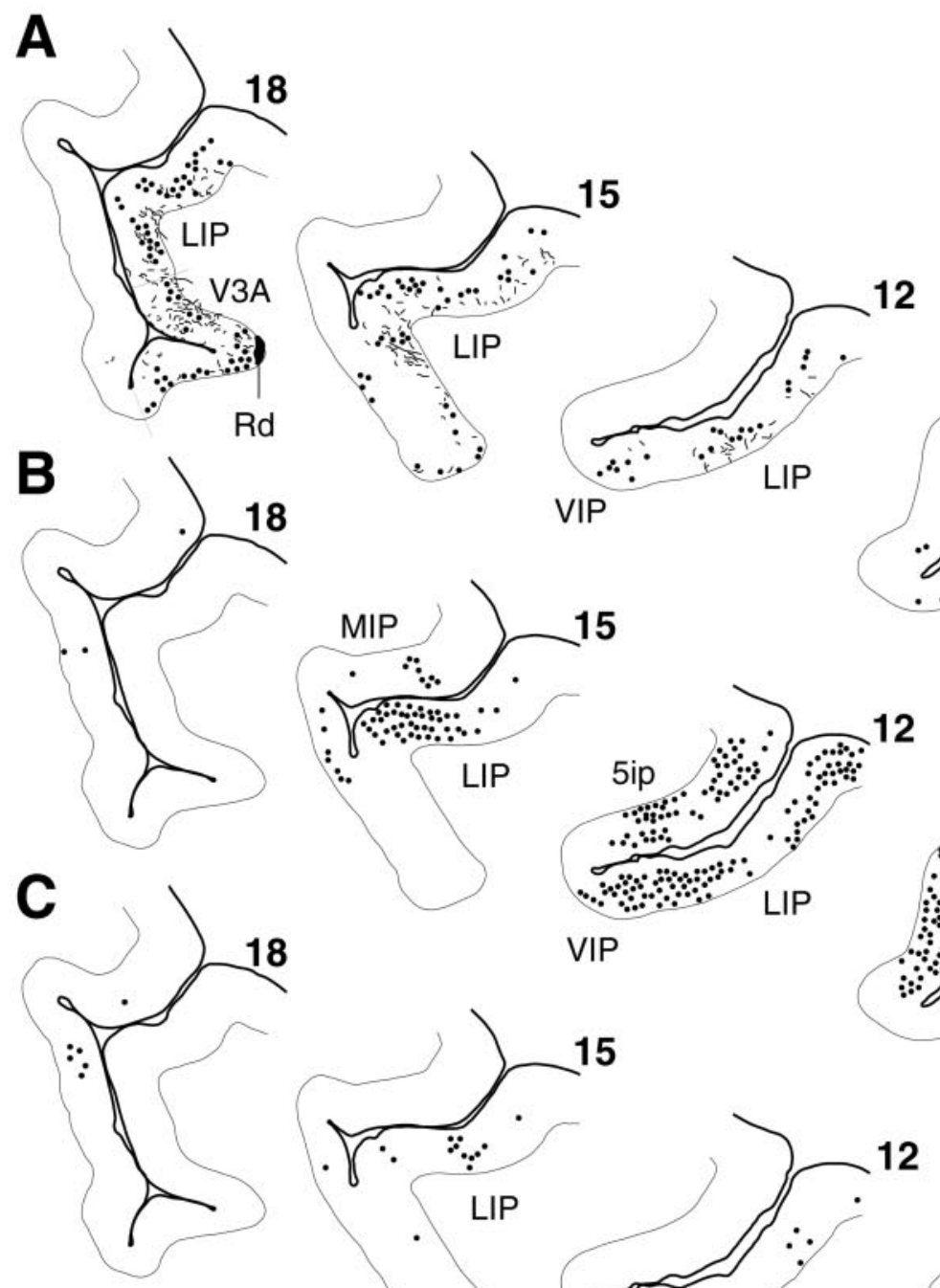

12
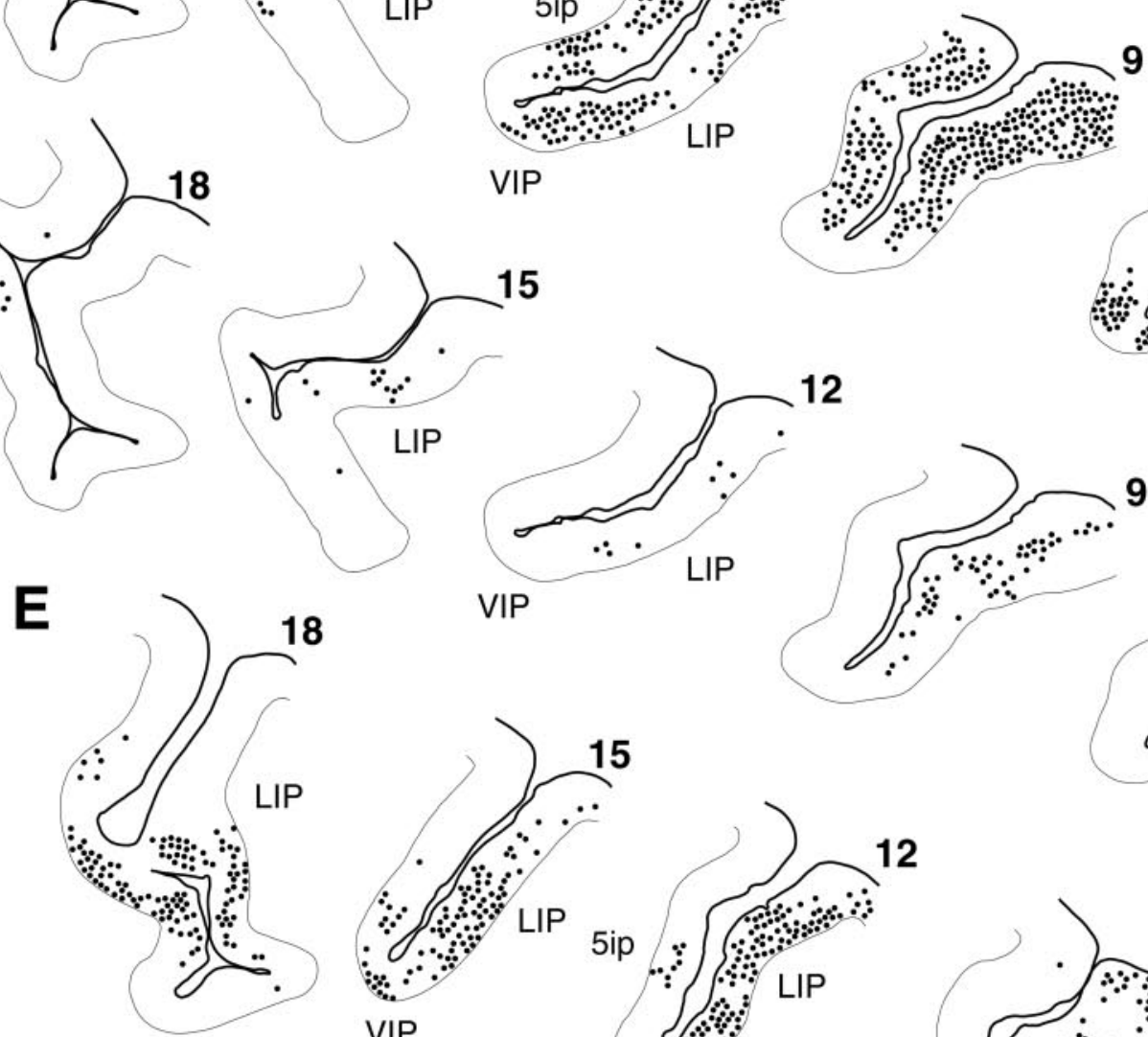

8 VIP

12
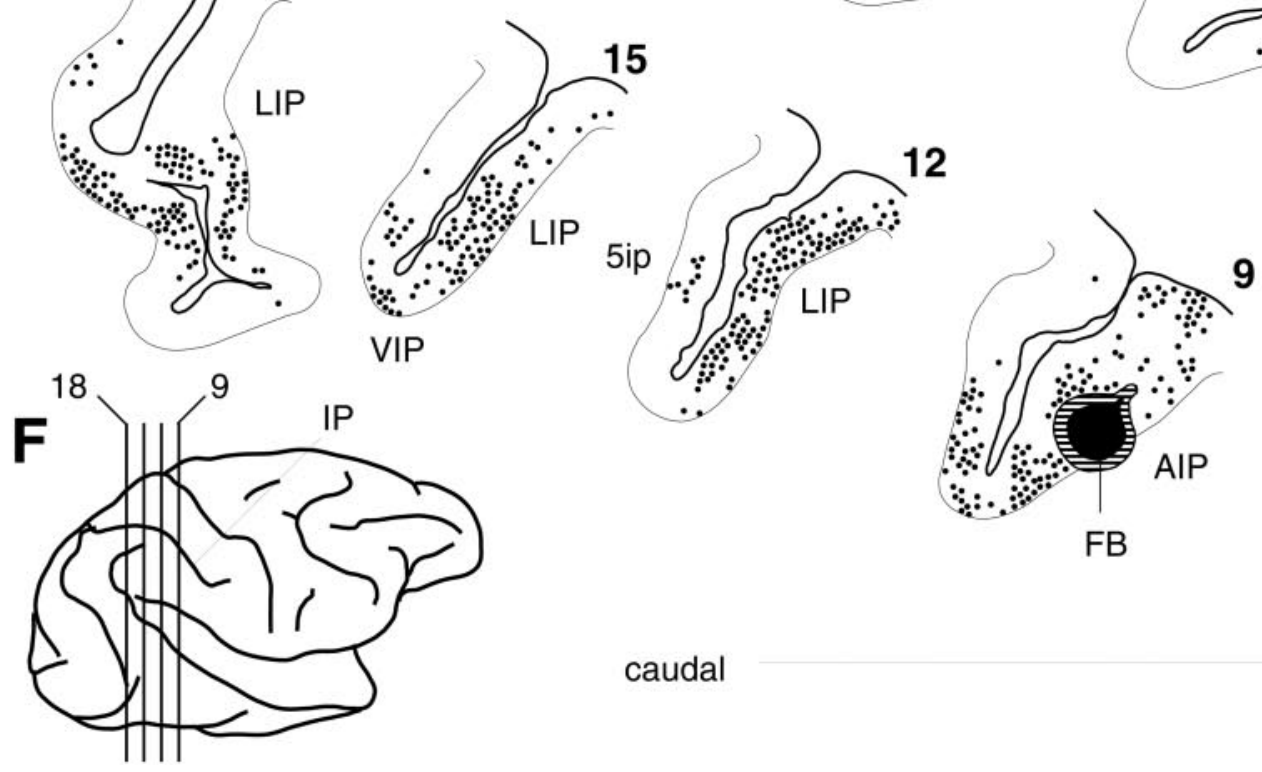

caudal

rostral 

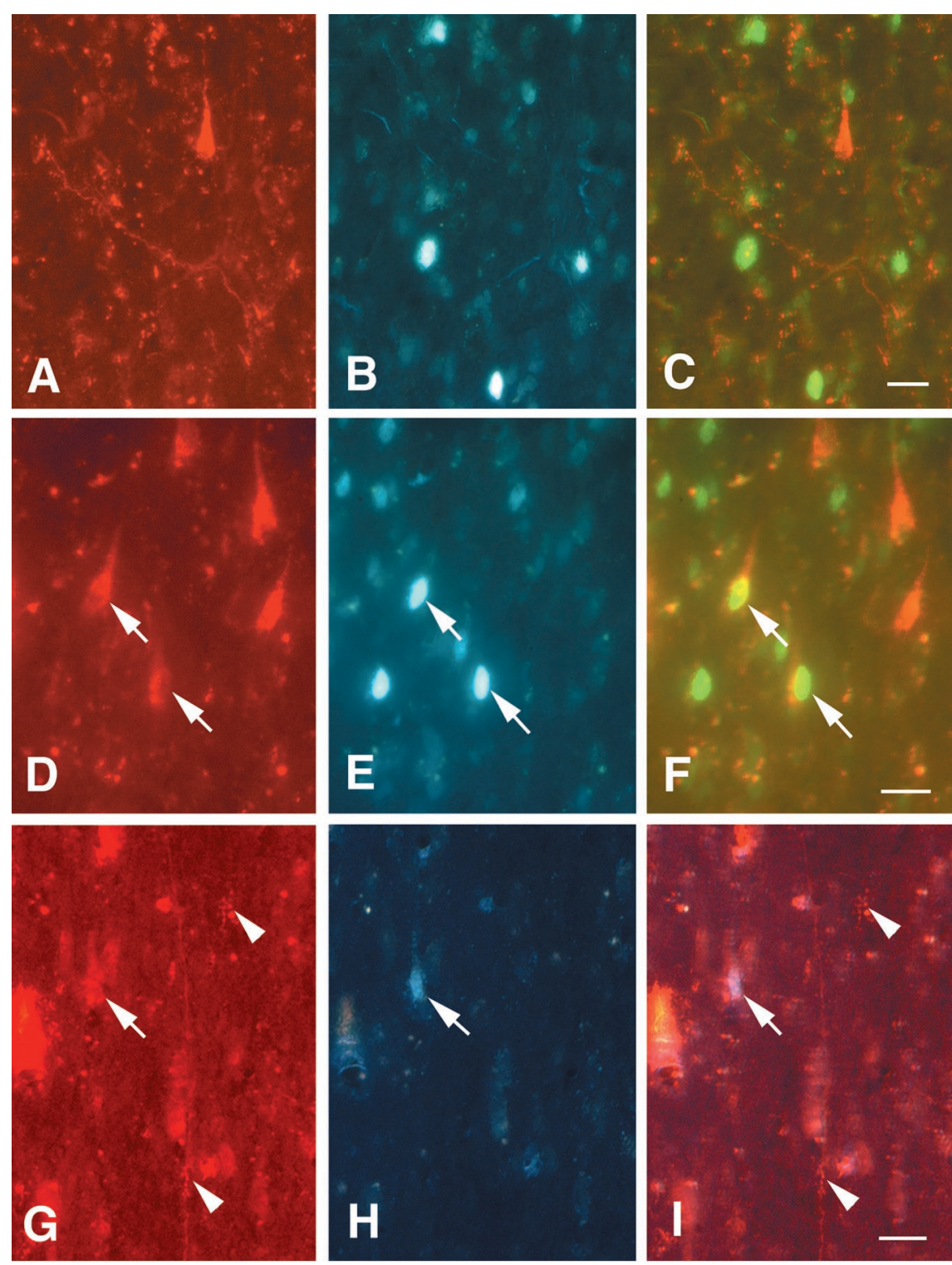

Figure 9. Photomicrographs of the posterior part of LIP after injecting a bidirectional fluorescent tracer $\mathrm{Rd}$ into the visual area V3A and retrograde fluorescent tracers NY and FB into AIP ipsilateral and contralateral, respectively, to the $\mathrm{Rd}$ injection. $A, \mathrm{~A}$ fiber and a neuron in layer 3 of LIP labeled with Rd injected into V3A. $B$, LIP neurons retrogradely labeled with NY injected into AIP in the same field as that of $A$. $C$, A merged photograph of $A$ and $B . D$, Rd-labeled neurons in layer 3. $E$, NY-labeled neurons. $F$, A merged photograph of $D$ and $E$ showing double-labeled neurons (arrows). $G$, Rd-labeled neurons and fibers with varicosities and terminal-like swellings (arrowheads) in LIP ipsilateral to Rd injection. $H$, A neuron in LIP labeled with FB injected into the contralateral AIP in the same field as that of $G$. I, A merged photograph of $G$ and $H$ showing a double-labeled LIP neuron (arrow) that projected to ipsilateral V3A and contralateral AIP, in the vicinity of labeled fibers and terminals (arrowheads). Scale bars, $20 \mu \mathrm{m}$.
The injection of FB was confined to AIP contralateral to the V3A injection and covered layers 3-6. Because layer 4 is the main target of forward corticocortical projection neurons in most cortical areas (Maunsell and Van Essen, 1983; Van Essen and Maunsell, 1983; Felleman and Van Essen, 1991), the limited laminar extent of this injection probably did not result in any missed projections from LIP to contralateral AIP. The tracer labeled the connections similar to those found in other cases in which the injection site involved all layers of the cortex. Although the injection spread to the white matter that was adjacent to layer 6 , the needle track did not, thus suggesting that no artifactual labeling resulted. The injection thus probably labeled all cortical connections. FB-labeled commissural neurons were distributed mainly in layers 3 and 5 of LIP and in layer 3 of MIP (Fig. 8C). The FB-labeled neurons in the posterior part of LIP that projected to the contralateral AIP were also found in the vicinity of the fibers and terminal arbors labeled with Rd (Fig. 9G-I). Some of the FB-labeled neurons projecting to the contralateral AIP were also labeled with Rd and thus projected to V3A (Fig. 9G-I). On the side of the intraparietal areas contralateral to the $\mathrm{Rd}$

$\leftarrow$

Figure 8. Distribution of labeling in the intraparietal areas demonstrating the overlap in the lateral intraparietal area $(L I P)$ of anterograde labeling from the visual area $V 3 A$ and retrograde labeling ipsilaterally and contralaterally from the anterior intraparietal area $(A I P)$. Tetramethylrhodamine dextran $(R d)$ was injected into $V 3 A(A)$, Nuclear Yellow $(N Y)$ was injected into the ipsilateral $A I P(B)$, and Fast blue $(F B)$ was injected into the contralateral $A I P(E)$. A, After the injection of $R d$ into $V 3 A$, labeled fibers with terminals (lines) and neurons ( filled circles) were distributed in the entire rostrocaudal LIP. B, After the injection of $N Y$ into $A I P$, retrogradely labeled neurons ( filled circles) were distributed in $A I P, L I P$, the intraparietal part of area 5 (5ip), the ventral intraparietal area $(V I P)$, and the medial intraparietal area $(M I P)$. $C$, After the injection of $F B$ into the left $A I P$ contralateral to the $R d$ injection, retrogradely labeled neurons ( filled circles) were distributed in the right $A I P, L I P$, and $M I P$. D, Drawing of the hemisphere showing the levels of the frontal sections in $A-C$. E, Injection of $F B$ into $A I P$ contralateral to the Rd injection. Retrogradely labeled neurons ( filled circles) were distributed in $A I P, L I P, 5 i p, V I P$, and MIP. F, Drawing of the hemisphere showing the levels of the frontal sections in $E$. $E$ and $F$ are flipped horizontally. The conventions are the same as those in Figure 4. 


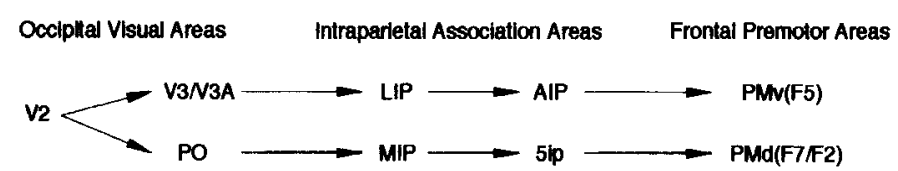

Figure 10. Schema of parallel pathways conveying visual information for hand movements through the intraparietal areas. 5ip, Intraparietal part of area $5 ; A I P$, anterior intraparietal area; $L I P$, lateral intraparietal area; $M I P$, medial intraparietal area; $P M d$, dorsal premotor area $6 ; P M v$, ventral premotor area $6 ; P O$, parieto-occipital area; $V 2$, visual area $\mathrm{V} 2 ; \mathrm{V} 3 / \mathrm{V} 3 \mathrm{~A}$, visual area V3/V3A complex.

injection into V3A, FB-labeled association neurons were found in layers 3 and 5 of LIP, VIP, 5ip, and MIP (Fig. 8E). Very few FB-labeled commissural neurons were found in the anterior part of the medial bank of the intraparietal sulcus.

\section{DISCUSSION}

We demonstrated the neuronal connections from V3A to LIP and then from LIP to AIP in the lateral bank of the intraparietal sulcus that may underlie the visuomotor transformation from 3-D object vision to prehensile hand movements. This circuit parallels the connections in the medial bank of the intraparietal sulcus, from PO (V6/V6A) to MIP, and PO and MIP to 5ip or PEa, which also underlie hand movements (Johnson et al., 1993, 1996; Luppino et al., 1993; Caminiti et al., 1998, 1999; Shipp et al., 1998; Burnod et al., 1999). Two parallel visual pathways for hand movements thus exist in the parietal association areas lining the intraparietal sulcus: one is in the medial bank and another is in the lateral bank of the intraparietal sulcus (Fig. 10).

\section{Myeloarchitecture and Cat-301 immunostaining of the intraparietal areas}

We confirmed the previous reports on the characteristics of myelin staining for areas LIP, VIP, PIP, V3A, and 5 (Seltzer and Pandya, 1980; Blatt et al., 1990; Preuss and Goldman-Rakic, 1991) and added some observation on the myelin structure of area AIP. We also confirmed that Cat-301 antibody is helpful in delineating the borders of the areas relating to the M pathway, such as V3, MIP, VIP, and LIP (Hockfield et al., 1983; McGuire et al., 1989; DeYoe et al., 1990), and further showed that it is useful in distinguishing AIP from the other parietal areas.

\section{The connections of V3A, LIP, and AIP}

The present results confirmed the reciprocal connections between V3A and LIP. V3A occupies the rostral part of the dorsomedial area of Beck and Kaas (1999) and receives major inputs from V2 (Gattas et al., 1997; Beck and Kaas, 1999) and V3 (Felleman et al., 1997; Beck and Kaas, 1999) as well as sparse inputs from V1 (Zeki, 1978, 1980; Van Essen et al., 1986; Beck and Kaas, 1999). V3A also receives inputs from the middle temporal area (Ungerleider and Desimone, 1986; Beck and Kaas, 1999), the middle superior temporal area (Boussaoud et al., 1990; Beck and Kaas, 1999), the fundus of the superior temporal visual area (Boussaoud et al., 1990; Beck and Kaas, 1999), and PO (Colby et al., 1988; Beck and Kaas, 1999). V3A has substantial reciprocal connections with LIP (Cavada and Goldman-Rakic, 1989; Andersen et al., 1990a; Blatt et al., 1990; Morel and Bullier, 1990; Baizer et al., 1991); V3A thus provides main visual inputs to LIP. Accordingly, the neurons in V3A show visual activity that is modulated by gaze (Galletti and Battaglini, 1989; Nakamura and Colby, 2000), and a similar modulation is also observed in the neurons of
LIP (Andersen and Mountcastle, 1983; Andersen et al., 1985b, 1990b).

LIP was originally defined on the basis of its connections from the frontal eye field (Andersen et al., 1985a). LIP has been reported to be reciprocally connected with PO, V3, V3A, V4, middle superior temporal area, dorsal prelunate area, area 7a, medial dorsal parietal area, VIP, area 5, area 8a, and area 46 (Andersen et al., 1990a; Blatt et al., 1990). In addition to the reciprocal connections, LIP has been reported to receive projections from areas $7 \mathrm{~b}, 7 \mathrm{~m}$, and $24 \mathrm{a}$, and also sends axons to the middle temporal area, the posterior inferior temporal area TEO, and the inferior temporal area TE. LIP thus receives and integrates visual information of both space and objects from areas in the dorsal and ventral streams and somatosensory information from the somatosensory areas and sends the integrated information to the visuomotor and motor-related areas.

This is the first study to inject tracers into AIP. Previous knowledge about connections of AIP were limited to the report that neurons in AIP do not project to the frontal eye field but project to ventral premotor area 6 (Petrides and Pandya, 1984; Matelli et al., 1986; Johnson et al., 1996; Gallese et al., 1997).

\section{LIP relay of connections from V3A to AIP}

In contrast to our expectations, the results showed that the projections from V3A terminated throughout the rostrocaudal extent of LIP, and the neurons projecting to AIP were not confined to the posterior part of LIP but were distributed throughout LIP. As a result, visual information from V3A is sent to the entire LIP, and then from the entire LIP to AIP. This finding correlates with the report by Tian and Lynch (1996) in which a single small injection of a retrograde tracer into the frontal eye field labeled neurons throughout the rostrocaudal extent of LIP. In accordance with this finding, the present mapping of the recording sites also revealed that the 3 -D-responsive neurons were not restricted to the posterior part of the LIP. Moreover, because we only recorded the neuronal responses to the stimuli of $20-60^{\circ}$ in size, we did not record any neurons responding to 3 -D visual stimuli of objects smaller than $20^{\circ}$ or larger than $60^{\circ}$. The recordings may thus cover only a limited area of the $3-\mathrm{D}$ visually responsive region of the LIP. If so, the modules of the 3-D visual information processing for the hand manipulation may be distributed in a wider region of the LIP and thus may overlap the distribution of other modules such as those related to the saccadic eye movements. Interestingly, the distribution of neurons responding to the 3 -D visual stimuli was similar to the location of neurons related to the saccadic eye movements (Gnadt and Andersen, 1988; Barash et al., 1991a) and attention (Robinson et al., 1995), as well as to the distribution of neurons projecting to the intermediate layers of the superior colliculus (Lynch et al., 1985) and to the frontal eye fields (Tian and Lynch, 1996).

\section{Functional consideration of LIP}

Neurons in LIP are involved in visuomotor integration functions (Andersen, 1987, 1995; Andersen and Zipser, 1988; Andersen et al., 1992), and are related not only to saccade and saccade preparation (Mountcastle et al., 1975; Lynch et al., 1977; Robinson et al., 1978; Gnadt and Andersen, 1988; Andersen 1989; Barash et al., 1991a,b; Andersen et al., 1992; Duhamel et al., 1992; Goldberg and Colby, 1992; Lynch, 1992) but also to visual response (Yin and Mountcastle, 1977; Robinson et al., 1978; Bushnell et al., 1981; Andersen et al., 1987; Colby et al., 1996). Some neurons in LIP respond to the saccades in 3-D space (Gnadt and 
Mays, 1995) and thus are related to 3-D space vision. LIP is also related to several aspects of spatial information processing such as attention (Lynch et al., 1977; Robinson et al., 1978, 1995; Bushnell et al., 1981; Mountcastle et al., 1981, 1987; Lynch and McLaren, 1989; Goldberg et al., 1990; Colby et al., 1996; Gottlieb et al., 1998; Corbetta et al., 2000), intention (Andersen, 1995; Bracewell et al., 1996; Mazzoni et al., 1996; Snyder et al., 1997), and anticipation (Colby et al., 1993b; Eskandar and Assad, 1999) and thus is suggested to be related in a more general role in visuospatial behavior (Colby et al., 1996).

In the present study, we focused on 3-D visual responses and their relation to hand manipulation; however, it should also be pointed out that neurons in LIP are also active during reaching hand movements. Mountcastle and colleagues (1975) reported that $24.7 \%$ of 474 neurons in area 7 (mostly in LIP) were reach and hand manipulation neurons. They classified 51 of $474(10.8 \%)$ neurons as hand manipulation and 61 of $474(12.9 \%)$ neurons as reach. They also classified, in area 5, 53 of $977(3.4 \%)$ neurons as hand manipulation and 52 of 977 (5.3\%) neurons as reach (Mountcastle et al., 1975). This is in accordance with our results that neurons in the MIP were also responsive to 3-D visual stimuli. As was the case in our study, in area 7, much overlap has been reported in the sites at which visual, visuomotor, fixation, and reach/hand manipulation neurons were isolated (Hyvarinen and Poranen, 1974; Mountcastle et al., 1975; Hyvarinen and Shelepin, 1979; Leinonen et al., 1979).

The LIP neurons are related not only to visual information processing but also to motion signal generation (Hyvarinen and Poranen, 1974; Mountcasle et al., 1975; Hyvarinen and Shelepin, 1979). Approximately one-fourth of all the neurons recorded in area 7, mostly in LIP, reported by Mountcastle et al. (1975) and one-third of neurons in area 7 reported by Hyvarinen and Poranen (1974) were manipulation neurons associated with reaching and hand movement. Because the hand motor neurons of the parietal cortex were neither sensory nor motor in nature, Mountcastle et al. (1975) suggested the "command hypothesis," i.e., the parietal neurons have command functions for manual exploration of surrounding space. The posterior parietal cortex not only reacts to sensory stimuli but also participates in the guidance of voluntary motor acts and integrates sensory and intentional factors (Hyvarinen, 1982). As in LIP, the responses of neurons in AIP are visual (Sakata et al., 1995), visual memory related (Murata et al., 1996), and motor (Gallese et al., 1994) in the grasp-related behavior. LIP and AIP therefore may relate visual and hand motor integration and motor command functions through the connections between these areas.

\section{REFERENCES}

Andersen RA (1987) Inferior parietal lobule function in spatial perception and visuomotor integration. In: The handbook of physiology. Section 1: the nervous system, Vol IV. Higher functions of the brain, Part 2 (Plum F, Mountcastle VB, Geiger ST, eds), pp 483-518. Bethesda, MD: American Physiological Society.

Andersen RA (1989) Visual and eye movement functions of the posterior parietal cortex. Annu Rev Neurosci 12:377-403.

Andersen RA (1995) Encoding of intention and spatial location in the posterior parietal cortex. Cereb Cortex 5:457-469.

Andersen RA, Mountcastle VB (1983) The influence of the angle of gaze upon the excitability of the light-sensitive neurons of the posterior parietal cortex. J Neurosci 3:532-548.

Andersen RA, Zipser D (1988) The role of posterior parietal cortex in coordinate transformations for visual-motor integration. Can J Physiol Pharmacol 66:488-501.

Andersen RA, Asanuma C, Cowan WM (1985a) Callosal and prefrontal associational projecting cell populations in area $7 \mathrm{a}$ of the macaque monkey: a study using retrogradely transported fluorescent dyes. J Comp Neurol 232:443-455.
Andersen RA, Essik GK, Siegel RM (1985b) Encoding of spatial location by posterior parietal neurons. Science 230:456-458.

Andersen RA, Essick GK, Siegel RM (1987) Neurons of area 7 activated by both visual stimuli and oculomotor behavior. Exp Brain Res 67:316-322.

Andersen RA, Asanuma C, Essick G Siegel RM (1990a) Corticocortical connections of anatomically and physiologically defined subdivisions within the inferior parietal lobule. J Comp Neurol 296:65-113.

Andersen RA, Bracewell RM, Barash S, Gnadt JW, Foggassi L (1990b) Eye position effects on visual, memory, and saccade-related activity in areas LIP and 7a of macaque. J Neurosci 10:1176-1196.

Andersen RA, Brotchie PR, Mazzoni P (1992) Evidence for the lateral intraparietal area as the parietal eye field. Curr Opin Neurobiol $2: 840-846$

Baizer JS, Ungerleider LG, Desimone R (1991) Organization of visual inputs to the inferior temporal and posterior parietal cortex in macaques. J Neurosci 11:168-190.

Barash S, Bracewell RM, Fogassi L, Gnadt JW, Andersen RA (1991a) Saccade-related activity in the lateral intraparietal area. I. Temporal properties; comparison with area 7a. J Neurophysiol 66:1095-1108.

Barash S, Bracewell RM, Fogassi L, Gnadt JW, Andersen RA (1991b) Saccade-related activity in the lateral intraparietal area. II. Spatial properties. J Neurophysiol 66:1109-1124.

Beck PD, Kaas JH (1999) Cortical connections of the dorsomedial visual area in old world macaque monkeys. J Comp Neurol 406:487-502.

Blatt GJ, Andersen RA, Stoner GR (1990) Visual receptive field organization and cortico-cortical connections of the lateral intraparietal area (area LIP) in the macaque. J Comp Neurol 299:421-445.

Boussaoud D, Ungerleider LG, Desimone R (1990) Pathway for motion analysis: cortical connections of the medial superior temporal and fundus of the superior temporal visual areas in the macaque. J Comp Neurol 296:462-495.

Bracewell RM, Mazzoni P, Barash S, Andersen RA (1996) Motor intention activity in the macaque's lateral intraparietal area. II. Changes of motor plan. J Neurophysiol 76:1457-1464.

Burnod Y, Baraduc P, Battaglia-Mayer A, Guigon E, Koechlin E, Ferraina S, Lacquaniti F, Caminiti R (1999) Parieto-frontal coding of reaching: an integrated framework. Exp Brain Res 129:325-346.

Bushnell MC, Goldberg ME, Robinson DL (1981) Behavioral enhancement of visual responses in monkey cerebral cortex. I. Modulation in posterior parietal cortex related to selective visual attention. J Neurophysiol 46:755-772.

Caminiti R, Ferraina S, Mayer AB (1998) Visuomotor transformations: early cortical mechanisms of reaching. Curr Opin Neurobiol 8:753-761.

Caminiti R, Genovesio A, Marconi B, Mayer AB, Onorati P, Ferraina S, Motsuda T, Giannetti S, Squatrito S, Maioli MG, Molinari M (1999) Early coding of reaching: frontal and parietal association connections of parieto-occipital cortex. Eur J Neurosci 11:3339-3345.

Carroll EW, Wong-Riley MTT (1984) Quantitative light and electron microscopic analysis of cytochrome oxidase-rich zones in the striate cortex of the squirrel monkey. J Comp Neurol 222:1-17.

Cavada C, Goldman-Rakic PS (1989) Posterior parietal cortex in rhesus monkey. I. Parcellation of areas based on distinctive limbic and sensory corticocortical connections. J Comp Neurol 287:393-421.

Colby CL, Gattass R, Olson CR, Gross CG (1988) Topographical organization of cortical afferents to extrastriate visual area PO in the macaque: a dual tracer study. J Comp Neurol 269:392-413.

Colby CL, Duhamel JR, Goldberg ME (1993a) Ventral intraparietal area of the macaque: anatomic location and visual response properties. J Neurophysiol 69:902-914.

Colby CL, Duhamel JR, Goldberg ME (1993b) The analysis of visual space by the lateral intraparietal area of the monkey: the role of extraretinal signals. In: Progress in brain research, Vol 95 (Hicks TP, Molotchnikoff S, Ono T, eds), pp 307-316. Amsterdam: Elsevier.

Colby CL, Duhamel JR, Goldberg ME (1996) Visual, presaccadic, and cognitive activation of single neurons in monkey lateral intraparietal area. J Neurophysiol 76:2841-2852.

Corbetta M, Kincade JM, Ollinger JM, McAvoy MP, Shulman GL (2000) Voluntary orienting is dissociated from target detection in human posterior parietal cortex. Nat Neurosci 3:292-297.

DeYoe EA, Hockfield S, Garren H, Van Essen DC (1990) Antibody labeling of functional subdivisions in visual cortex: Cat-301 immunoreactivity in striate and extrastriate cortex of the macaque monkey. Vis Neurosci 5:67-81.

Duhamel JR, Colby CL, Goldberg ME (1992) The updating of the representation of visual space in parietal cortex by intended eye movements. Science 255:90-92

Endo K, Haranaka Y, Adams DL, Kusunoki M, Sakata H (1998) Effects of different types of disparity cues on the response of axis-orientation selective (AOS) cells in the monkey parietal cortex. Soc Neurosci Abstr 24:1397.

Eskandar EN, Assad JA (1999) Dissociation of visual, motor and predictive signals in parietal cortex during visual guidance. Nat Neurosci 2:88-93.

Felleman DJ, Van Essen DC (1987) Receptive field properties of neu- 
rons in area V3 of macaque monkey extrastriate cortex. J Neurophysiol 57:889-920.

Felleman DJ, Van Essen DC (1991) Distributed hierarchical processing in the primate cerebral cortex. Cereb Cortex 1:1-47.

Felleman DJ, Burkhalter A, Van Essen DC (1997) Cortical connections of areas V3 and VP of macaque monkey extrastriate visual cortex. J Comp Neurol 379:21-47.

Gallese V, Murata A, Kaseda M, Niki N, Sakata H (1994) Deficit of hand preshaping after muscimol injection in monkey parietal cortex. NeuroReport 5:1525-1529.

Gallese V, Fadiga L, Fagassi L, Luppino G, Murata A (1997) A parietofrontal circuit for hand grasping movements in the monkey: evidence from reversible inactivation experiments. In: Parietal lobe contributions to orientation in 3D space (Thier P, Karnath HO, eds), pp 619-630. Heidelberg: Springer.

Galletti C, Battaglini PP (1989) Gaze-dependent visual neurons in area V3A of monkey prestriate cortex. J Neurosci 9:1112-1125.

Gallyas F (1979) Silver staining of myelin by means of physical development. Neurol Res 1:203-209.

Gattass R, Sousa APB, Gross CG (1988) Visuotopic organization and extent of V3 and V4 of the macaque. J Neurosci 8:1831-1945.

Gattass R, Sousa APB, Mishkin M, Ungerleider LG (1997) Cortical projections of area V2 in the macaque. Cereb Cortex 7:110-129.

Gnadt JW (2000) Neural codes for three-dimensional space. In: Accommodation and vergence mechanisms in the visual system (Franzen $\mathrm{O}$, Richer H, Stark L, eds), pp 11-19. Basel: Birkhauser Verlag.

Gnadt JW, Andersen RA (1988) Memory related motor planning activity in posterior parietal cortex of macaque. Exp Brain Res 70:216-220.

Gnadt JW, Beyer J (1998) Eye movements in depth: what does monkey's parietal cortex tell the superior colliculus? NeuroReport 9:233-238.

Gnadt JW, Mays LE (1995) Neurons in monkey parietal area LIP are tuned for eye-movement parameters in three-dimensional space. J Neurophysiol 73:280-297.

Goldberg ME, Colby CL (1992) Oculomotor control and spatial processing. Curr Opin Neurobiol 2:198-202.

Goldberg ME, Colby CL, Duhamel JR (1990) Representation of visuomotor space in the parietal lobe of the monkey. Cold Spring Harbor Symp Quant Biol 105:729-739.

Goodale MA, Milner AD (1992) Separate visual pathways for perception and action. Trends Neurosci 15:20-25.

Goodale MA, Murphy KJ (1997) Action and perception in the visual periphery. In: Parietal lobe contributions to orientation in 3D space (Their P, Karnath H-O, eds), pp 447-461. Heidelberg: Springer.

Goodale MA, Milner AD, Jakobson LS, Carey DP (1991) A neurological dissociation between perceiving objects and grasping them. Nature 349:154-156.

Gottlieb JP, Kusunoki M, Goldberg ME (1998) The representation of visual salience in monkey parietal cortex. Nature 391:481-484.

Hendry SHC, Hockfield S, Jones EG, McKay RDG (1984) Monoclonal antibody that identifies subsets of neurons in the central visual system of monkey and cat. Nature 307:267-269.

Hendry SHC, Jones EG, Hockfield S, McKay RDG (1988) Neuronal populations stained with the monoclonal antibody Cat-301 in the mammalian cerebral cortex and thalamus. J Neurosci 8:518-542.

Hockfield S, McKay RDG, Hendry SHC, Jones EG (1983) A surface antigen that identifies ocular dominance columns in the visual cortex and laminar features of the lateral geniculate nucleus. Cold Spring Harbor Symp Quant Biol 48:877-889.

Hof PR, Morrison JH (1995) Neurofilament protein defines regional patterns of cortical organization in the macaque monkey visual system: a quantitative immunohistochemical analysis. J Comp Neurol 352:161-186.

Hyvarinen J (1982) Posterior parietal lobe of the primate brain. Physiol Rev 62:1060-1129.

Hyvarinen J, Poranen A (1974) Function of the parietal associative area 7 as revealed from cellular discharges in alert monkeys. Brain 97:673-692.

Hyvarinen J, Shelepin Y (1979) Distribution of visual and somatic functions in the parietal associative area 7 of the monkey. Brain Res 169:561-564.

Jeannerod M, Arbib MA, Rizzolatti G, Sakata H (1995) Grasping objects: the cortical mechanisms of visuomotor transformation. Trends Neurosci 18:314-320.

Johnson PB, Ferraina S, Caminiti R (1993) Cortical networks for visual reaching. Exp Brain Res 97:361-365.

Johnson PB, Ferraina S, Bianchi L, Caminiti R (1996) Cortical networks for visual reaching: physiological and anatomical organization of frontal and parietal lobe arm regions. Cereb Cortex 6:102-119.

Johnson PB, Ferraina S, Garasto MR, Battaglia-Mayer A, Ercolani L, Burnod Y, Caminiti R (1997) From vision to movement: corticocortical connections and combinatorial properties of reaching-related neurons in parietal areas V6 and V6A. In: Parietal lobe contributions to orientation in 3D space (Their P, Karnath O, eds), pp 221-236. Heidelberg: Springer.
Kalaska JF, Scott SH, Cisek P, Sergio LE (1997) Cortical control of reaching movements. Curr Opin Neurobiol 7:849-859.

Kurata K (1991) Corticocortical inputs to the dorsal and ventral aspects of the premotor cortex of macaque monkeys. Neurosci Res 12:263-280.

Lacquaniti F, Guigon E, Bianchi L, Ferraina S, Caminiti R (1995) Representing spatial information for limb movement: role of area 5 in the monkey. Cereb Cortex 5:391-409.

Leinonen L, Hyvarinen J, Nyman G, Linnankoski I (1979) I. Functional properties of neurons in lateral part of associative area 7 in awake monkeys. Exp Brain Res 34:299-320.

Luppino G, Matelli M, Camarda R, Rizzolatti G (1993) Cortico-cortical connections of area F3 (SMA-proper) and area F6 (pre-SMA) in the macaque monkey. J Comp Neurol 338:114-140.

Lynch JC (1992) Saccade initiation and latency deficits after combined lesions of the frontal and posterior eye fields in monkeys. J Neurophysiol 68:1913-1916.

Lynch JC, McLaren JW (1989) Deficits of visual attention and saccadic eye movements after lesions of parieto-occipital cortex in monkeys. J Neurophysiol 61:74-90.

Lynch JC, Mountcastle VB, Talbot WH, Yin TCT (1977) Parietal lobe mechanisms for directed visual attention. J Neurophysiol 40:362-389.

Lynch JC, Graybiel AM, Lobeck LJ (1985) The differential projection of two cytoarchitectonic subregions of the inferior parietal lobule of macaque upon the deep layers of the superior colliculus. J Comp Neurol 235:241-254.

Matelli M, Luppino G, Rizzolatti G (1985) Patterns of cytochrome oxidase activity in the frontal agranular cortex of macaque monkey. Behav Brain Res 18:125-137.

Matelli M, Camarda R, Glickstein M, Rizzolatti G (1986) Afferent and efferent projections of the inferior area 6 in the macaque monkey. J Comp Neurol 251:281-298.

Matelli M, Govoni P, Galletti C, Kutz DF, Luppino G (1998) Superior area 6 afferents from the superior parietal lobule in the macaque monkey. J Comp Neurol 402:327-352.

Maunsell JHR, Van Essen DC (1983) The connections of the middle temporal visual area (MT) and their relationship to a cortical hierarchy in the macaque monkey. J Neurosci 3:2563-2586.

Mazzoni P, Bracewell RM, Barash S, Andersen RA (1996) Motor intention activity in the macaque's lateral intraparietal area. I. Dissociation of motor plan from sensory memory. J Neurophysiol 76:1439-1456.

McGuire PK, Hockfield S, Goldman-Rakic PS (1989) Distribution of Cat-301 immunoreactivity in the frontal and parietal lobes of the macaque monkey. J Comp Neurol 288:280-296.

Mesulum MM (1978) Tetramethyl benzidine for horseradish peroxidase neurohistochemistry: a non-carcinogenic blue reaction-product with superior sensitivity for visualizing neural afferents and efferents. J Histochem Cytochem 26:106-117.

Mesulum MM (1982) Tracing neural connections with horseradish peroxidase. New York: Wiley.

Milner AD, Goodale MA (1993) Visual pathway to perception and action. In: Progress in brain research, Vol 95 (Hicks TP, Molotchnikoff S, Ono T, eds), pp 317-337. Amsterdam: Elsevier.

Morel A, Bullier J (1990) Anatomical segregation of two cortical visual pathways in the macaque monkey. Vis Neurosci 4:555-578.

Mountcastle VB, Lynch JC, Georgopoulos A, Sakata H, Acuna C (1975) Posterior parietal association cortex of the monkey: command functions for operations in extrapersonal space. J Neurophysiol 38:871-908.

Mountcastle VB, Andersen RA, Motter BC (1981) The influence of attentive fixation upon the excitability of the light-sensitive neurons of the posterior parietal cortex. J Neurosci 1:1218-1235.

Mountcastle VB, Motter BC, Sternmetz MA, Sestokas AK (1987) Common and differential effects of attentive fixation on the excitability of parietal and prestriate (V4) cortical visual neurons in the macaque monkey. J Neurosci 7:2239-2255.

Murata A, Gallese V, Kaseda M, Sakata H (1996) Parietal neurons related to memory-guided hand manipulation. J Neurophysiol 75:2180-2186.

Murata A, Fediga L, Fagassi L, Gallese V, Raos V, Rizzolatti G (1997) Object representation in the ventral premotor cortex (area F5) of the monkey. J Neurophysiol 78:2226-2230.

Murata A, Gallese V, Luppino G, Kaseda M, Sakata H (2000) Selectivity for the shape, size, and orientation of objects for grasping in neurons of monkey parietal area AIP. J Neurophysiol 83:2580-2601.

Nakamura K, Colby CL (2000) Visual, saccade-related, and cognitive activation of single neurons in monkey extrastriate area V3A. J Neurophysiol 84:677-692.

Pandya DN, Seltzer B (1982) Intrinsic connections and architectonics of posterior parietal cortex in the rhesus monkey. J Comp Neurol 204:196-210

Petrides M, Pandya DN (1984) Projections to the frontal cortex from the posterior parietal region in the rhesus monkey. J Comp Neurol 228:105-116.

Preuss TM, Goldman-Rakic PS (1991) Architectonics of the parietal and temporal association cortex in the strepsirhine primate Galago com- 
pared with the anthropoid primate Macaca. J Comp Neurol 310:475-506.

Rizzolatti G, Camarda R, Fogassi L, Gentilucci M, Luppino G, Matelli M (1988) Functional organization of inferior area 6 in the macaque monkey. II. Area F5 and the control of distal movements. Exp Brain Res 71:491-507.

Rizzolatti G, Gentilucci M, Camarda RM, Gallese V, Luppino G, Matelli M, Fogassi L (1990) Neurons related to reaching-grasping arm movements in the rostral part of area 6 (area $6 \mathrm{a} \beta)$. Exp Brain Res 82:337-350.

Rizzolatti G, Fogassi L, Gallesse V (1997) Parietal cortex: from sight to action. Curr Opin Neurobiol 7:562-567.

Robinson DL, Goldberg ME, Stanton GB (1978) Parietal association cortex in the primate: sensory mechanisms and behavioral modulations. J Neurophysiol 41:910-932.

Robinson DL, Bowman EM, Kertzman C (1995) Covert orienting of attention in macaques. II. Contributions of parietal cortex. J Neurophysiol 74:698-712.

Rockland KS, Pandya DN (1979) Laminar origins and terminations or cortical connections of the occipital lobe in the rhesus monkey. Brain Res 179:3-20.

Sakata H, Taira M (1994) Parietal control of hand action. Curr Opin Neurobiol 4:847-856.

Sakata H, Taira M, Murata A, Mine S (1995) Neural mechanisms of visual guidance of hand action in the parietal cortex of the monkey. Cereb Cortex 5:429-438.

Seltzer B, Pandya DN (1980) Converging visual and somatic sensory cortical input to the intraparietal sulcus of the rhesus monkey. Brain Res 192:339-351.

Shikata E, Tanaka Y, Nakamura H, Taira M, Sakata H (1996) Selectivity of the parietal visual neurons in 3-D orientation of surface of stereoscopic stimuli. NeuroReport 7:2389-2394.

Shipp S, Blanton M, Zeki S (1998) A visuo-somatomotor pathway through superior parietal cortex in the macaque monkey: cortical connections of areas V6 and V6A. Eur J Neurosci 10:3171-3193.
Shu S, Ju G, Fan L (1988) The glucose oxidase-DAB-nickel method in peroxidase histochemistry of the nervous system. Neurosci Lett 85:169-171

Snyder LH, Batista AP, Andersen RA (1997) Coding of intention in the posterior parietal cortex. Nature 386:167-170.

Taira M, Mine S, Georgopoulos AP, Murata A, Sakata H (1990) Parietal cortex neurons of the monkey related to the visual guidance of hand movement. Exp Brain Res 83:29-36.

Tanne J, Boussaoud D, Boyer-Zeller N, Rouiller EM (1995) Direct visual pathways for reaching movements in the macaque monkey. NeuroReport 7:267-272.

Tian JR, Lynch JC (1996) Corticocortical input to the smooth and saccadic eye movement subregions of the frontal eye field in Cebus monkeys. J Neurophysiol 76:2754-2771.

Ungerleider LG, Desimone R (1986) Cortical connections of visual area MT in the macaque. J Comp Neurol 248:190-222.

Ungerleider LG, Mishkin M (1982) Two cortical visual systems. In: Analysis of visual behavior (Ingle DJ, Goodale MA, Mansfield RJW, eds), pp 549-586. Cambridge, MA: MIT.

Van Essen DC, Maunsell JHR (1983) Hierarchical organization and functional streams in the visual cortex. Trends Neurosci 6:370-375.

Van Essen DC, Newsome WT, Maunsell JHR, Bixby JL (1986) The projections from striate cortex (VI) to areas V2 and V3 in the macaque monkey: asymmetries, area boundaries and patchy connections. J Comp Neurol 244:451-480.

Vogt O, Vogt C (1919) Ergebnisse unerer Hirnforschung. J Psychol Neurol (Leipzig) 25:277-462.

von Bonin G, Bailey P (1947) The neocortex of Macaca mulatta. Urbana, IL: University of Illinois.

Yin TCT, Mountcastle VB (1977) Visual input to the visuomotor mechanisms of the monkey's parietal lobe. Science 197:1381-1383.

Zeki SM (1978) The third visual complex of rhesus monkey prestriate cortex. J Physiol (Lond) 277:245-272.

Zeki SM (1980) A direct projection from area V1 to area V3A of rhesus monkey visual cortex. Proc R Soc Lond B Biol Sci 207:499-506. 